\title{
Elucidation of Metabolic and Disposition Pathways for Maribavir in Nonhuman Primates through Mass Balance and Semi-Physiologically Based Modeling Approaches ${ }^{\mathrm{s}}$
}

\author{
Kefeng Sun and Devin Welty ${ }^{1}$ \\ Global Drug Metabolism and Pharmacokinetics, Takeda Development Center Americas, Inc., Lexington, Massachusetts
}

Received April 07, 2021; accepted August 26, 2021

\section{ABSTRACT}

Maribavir is in phase 3 clinical development for treatment of cytomegalovirus infection/disease in transplant recipients. Previous research conducted using only intact cynomolgus monkeys indicated biliary secretion as the primary elimination pathway for maribavir and that maribavir undergoes enterohepatic recirculation (EHR). To clarify the exact mechanisms of maribavir's EHR behavior, we studied its clearance pathways using intravenously administered ${ }^{14} \mathrm{C}$-labeled maribavir in intact and bile duct-cannulated (BDC) monkeys and constructed a semi-physiologically based pharmacokinetic (PBPK) model. Total radioactivity metabolite profiles in plasma and excreta were quantitatively determined along with plasma maribavir concentrations. Intact animals showed significantly lower clearance and longer half-lives in both total radioactivity and parent concentration in plasma than BDC monkeys. The primary in vitro and in vivo metabolic pathway for maribavir in monkey is direct glucuronidation; $\mathbf{N}$-dealkylation and renal clearance are minor pathways. In BDC monkeys, $73 \%$ of dose was recovered as maribavir glucuronides in bile, and $3 \%$ of dose was recovered as parent in bile and feces; in intact animals' feces, $\mathbf{5 8 \%}$ of dose was recovered as parent, and no glucuronides were detected. Therefore, EHR of maribavir occurs through biliary secretion of maribavir glucuronides, and this is followed by hydrolysis of glucuronides in the gut lumen and subsequent reabsorption of parent. A semi-PBPK model constructed from physiologic, in vitro, and in vivo BDC monkey data is capable of projecting maribavir's pharmacokinetic and EHR behavior in intact animals after intravenous or oral dosing and could be applied to modeling other xenobiotics that are subject to similar EHR processes.

\section{SIGNIFICANCE STATEMENT}

Through both mass balance and semi-physiologically based pharmacokinetic (semi-PBPK) modeling approaches, this study mechanistically and quantitatively elucidates maribavir's enterohepatic recirculation (EHR) behavior in monkeys, which occurs via extensive direct glucuronidation, biliary secretion of these glucuronides, luminal hydrolysis of glucuronides to parent, and subsequent reabsorption of the parent. The study also identifies important drug- and animal-specific parameters that determine the EHR kinetics, and the semi-PBPK model is readily applicable to other drugs that undergo similar metabolic and recirculation mechanisms.

\section{Introduction}

Cytomegalovirus (CMV) infection is a serious complication that frequently occurs in recipients of hematopoietic cell or solid organ transplantations (Teira et al., 2016; Hakimi et al., 2017). Treatment with the currently available antiviral therapies has limitations, such as drug toxicities and lack of efficacy against drug-resistant strains of CMV (Venton et al., 2014; Kotton et al., 2018). Maribavir (5,6-dichloro-2-(isopropylamino)-1, $\beta$-L-ribofuranosyl-1-H-benzimidazole; structure shown in Fig. 1) is a potent and

This research was funded by Takeda Development Center Americas, Inc.

K.S. is an employee of Takeda Development Center Americas, Inc. D.W. was an employee of Shire Human Genetic Therapies, Inc. (now Takeda Development Center Americas, Inc.) at the time of the study.

${ }^{1}$ Current affiliation: D.W. is currently an employee of Nuventra Pharma Sciences, Broomfield, Colorado.

https://dx.doi.org/10.1124/dmd.121.000493.

S This article has supplemental material available at dmd.aspetjournals.org. selective orally bioavailable benzimidazole riboside and is active against human CMV (Biron et al., 2002; Williams et al., 2003) by blocking nuclear egress of viral capsids through protein kinase UL97 inhibition (Krosky et al., 2003; Hamirally et al., 2009); this mechanism of action stands in contrast to that of DNA polymerase inhibitors (ganciclovir, valganciclovir, cidofovir, and foscarnet) and terminase inhibitor (letermovir) approved for management of CMV (Marty et al., 2017; Kotton et al., 2018). In two phase 2 studies, the majority of solid organ transplant and hematopoietic stem cell transplant recipients with CMV infection achieved viremia clearance after maribavir treatment across all doses studied [400, 800, and $1200 \mathrm{mg}$ twice daily (b.i.d.)]; maribavir exhibited comparable efficacy to valganciclovir, and recipients of maribavir experienced low incidences of neutropenia and renal toxicity (Maertens et al., 2019; Papanicolaou et al., 2019). The ongoing clinical development program for maribavir for the treatment of transplant recipients with CMV includes two phase 3 trials (NCT02927067, NCT02931539) (National Library of Medicine, 2020a,b).

The pharmacokinetics (PK), metabolism, and disposition of maribavir in nonclinical species have been previously reported (Koszalka et al., 2002).

ABBREVIATIONS: AUC, area under the curve; BDC, bile duct-cannulated; b.i.d., twice daily; BLQ, below the limit of quantitation; CMV, cytomegalovirus; EHR, enterohepatic recirculation; $f_{m}$, fraction metabolized; $f_{m}(G l u c)$, fraction metabolized by direct glucuronidation; Gl, gastrointestinal; GUS, $\beta$-glucuronidase; LC-MS/MS, liquid chromatography-tandem mass spectrometry; LSC, liquid scintillation counter; PBPK,

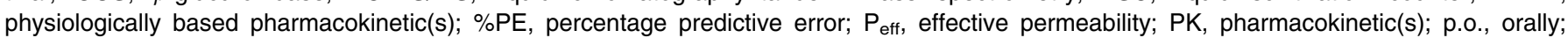
SITT, small intestine transit time; UGT, UDP-glucuronosyltransferase. 


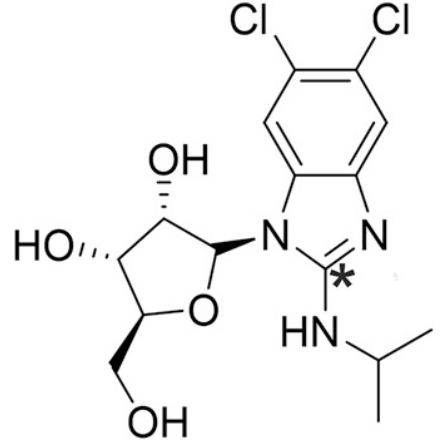

Fig. 1. Structure of maribavir (also known as TAK-620, SHP620, VP41263, and 1263 W94). The position of the ${ }^{14} \mathrm{C}$ atom is marked with an asterisk.

After intravenous or oral administration of ${ }^{14} \mathrm{C}$-labeled maribavir to both intact rat and monkey, a large proportion of radioactivity ( $\geq 89 \%$ in rat and up to $57 \%$ in monkey) was recovered as unchanged parent in feces, suggesting that biliary excretion may be the predominant route of elimination in these species. Renal clearance was thought to be a minor elimination pathway for maribavir, as indicated by the relatively low percentage dose recovered in urine, at $<6 \%$ total dose in rat and $<18 \%$ total dose in monkey; a portion of the radioactivity in urine was attributed to the $N$-dealkylated metabolite VP44469 [4\%-7\% total dose in both rat and monkey after intravenous or oral (p.o.) dosing] (Koszalka et al., 2002). VP44469 was also detected in feces of monkeys (11\% intravenous dose and $15.5 \%$ p.o. dose). Additionally, in these monkeys, a prolonged elimination phase was observed, suggesting that maribavir may undergo enterohepatic recirculation (EHR) before excretion. Biliary clearance was thus deemed the major clearance pathway and oxidative metabolism to VP44469 as the primary metabolic pathway, as indicated by parent and metabolite profiles in plasma, urine, and feces of intact animals (Koszalka et al., 2002).

Characterization of clearance pathways of xenobiotics is commonly evaluated using bile duct-cannulated (BDC) animals most often in rats but also larger animal species (Kimoto et al., 2017). The use of only intact animals in previous research (Koszalka et al., 2002) that pointed to biliary secretion being the primary elimination pathway for maribavir confounds determination of the mechanisms of its EHR. A lack of understanding on such mechanisms presents inherent uncertainty pertaining to evaluation of drug-drug interaction risks and factors driving interindividual variability in maribavir PK. Here we conducted additional definitive studies in monkeys, which were selected because of their relative closeness in physiology to humans, particularly in organs, such as liver and the gastrointestinal tract, and present quantitative evidence on the clearance pathways of maribavir in both intact and BDC monkeys as well as the mechanism of EHR in intact monkeys. We then constructed a semi-physiologically based pharmacokinetic (semi-PBPK) model and demonstrated that the PK of maribavir in intact monkeys could be projected with physiologically based modeling and simulations and also identified important parameters that drive the extent and kinetics of EHR of maribavir with implications in both nonhuman primates and humans.

\section{Materials and Methods}

\section{Materials}

Maribavir was provided by Carbogen AMCIS AG (Bubendorf, Switzerland). ${ }^{14} \mathrm{C}$-maribavir $(2.43 \mu \mathrm{Ci} / \mathrm{mg})$ and $\mathrm{d}_{7}$-maribavir were provided by Almac Sciences (Craigavon, Northern Ireland, United Kingdom). The chemical purity and radioactive purity of ${ }^{14} \mathrm{C}$-maribavir (lot SJJ-0004E-010-01) were $>98 \%$. Cryopreserved mixed-sex pooled human hepatocytes (20 donors) and cynomolgus monkey hepatocytes (6 donors) were purchased from BioreclamationIVT (Baltimore, MD). All buffers and chemicals used in in vitro studies were obtained from Sigma-Aldrich (St Louis, MO) or EMD Chemicals (Gibbstown, NJ).

\section{Animal Preparation and Dosing}

Animals. All animal housing and care conformed to the standards recommended by the Guide for the Care and Use of Laboratory Animals as adopted and promulgated by US National Institutes of Health and were approved by the Institution's Animal Care and Use Committee or local equivalent. Male cynomolgus monkeys were from the Covance (Princeton, NJ) stock colony. Animals were acclimated to study conditions for 5 weeks prior to dose administration. At dosing, animals weighed between 3.7 and $4.2 \mathrm{~kg}$ and were 3-4 years of age. Animals were assigned to either group 1 (intact, $n=3$ ) or group 2 (BDC, $n=3$ ). BDC surgery was done under sterile conditions using appropriate sedation and inhalation anesthetic. The bile duct was cannulated to allow collection of bile, and a second cannula was placed in the duodenum for the infusion of bile salts replacement solution or other fluids (Kimoto et al., 2017). Further details on animal husbandry and surgical procedures are provided in the Supplemental Material.

Dosing. ${ }^{14} \mathrm{C}-$ Maribavir dosing solution was prepared on the day of dosing. Animals were dosed with $13 \mathrm{mg} / \mathrm{kg}{ }^{14} \mathrm{C}$-maribavir (corresponding to a mean $31.5 \mu \mathrm{Ci} / \mathrm{kg}$; prepared in $7 \%$ ethanol and $15 \%$ propylene glycol in sterile saline vehicle) via intravenous infusion followed by a $2-\mathrm{ml}$ flush of saline. Details on the preparation and administration of ${ }^{14} \mathrm{C}$-maribavir are provided in the Supplemental Material.

\section{Pharmacokinetics and Excretion Balance}

Sample Collection. Blood was collected from both groups (intact and BDC) at predose and at 5, 15, and 30 minutes, and this was followed by 2, 6, 24, 48, $72,96,120,144$, and 168 hours postdose. At each time point, blood samples were collected from each animal for radioanalysis and metabolite profiling (1 $\mathrm{ml})$ and plasma extraction $(1.5 \mathrm{ml})$.

Blood samples were processed using standard techniques (Supplemental Material) and stored at $-70^{\circ} \mathrm{C}$ until analysis. Urine and feces from both groups and bile (group 2) were collected at 0-8 and 8-24 hours postdose and at 24-hour intervals through to at least 168 hours postdose. Nonbiologic samples (cage rinse, cage debris, cage wash, cage wipe, jacket extract, and urine wipe; bile duct cannulae for group 2) were also collected. Further details can be found in the Supplemental Material.

Radioactivity Measurement. Radioactivity in samples was measured using Model 2900TR and 2910TR liquid scintillation counters (LSCs; Packard Instrument Company, Downers Grove, IL) with Ultima Gold XR scintillation cocktail (PerkinElmer, Waltham, MA) for at least 5 minutes or 100,000 counts. Blood and fecal samples were further processed before analysis (see Supplemental Material). Other samples (including bile, urine, and plasma) were analyzed directly. To obtain disintegration-per-minute data, scintillation counting data (in cpm) were automatically corrected for counting efficiency using the external standardization technique, and an instrument-stored quench curve was generated from a series of sealed quenched standards. The representative lower limit of quantitation for radioactivity in blood and plasma was 40-ng equivalent/g for 72-hour samples in group 1, 24-hour samples in group 2, and 78-ng equivalent/g for all other blood and plasma samples.

\section{Metabolite Profiling}

Radioactivity Extraction Recovery. Extraction recoveries for each excreta were determined as described in the Supplemental Material.

Plasma. Plasma samples were pooled by group and at each time point. Radioactivity in each pooled sample was determined by LSC. Reconstituted samples (see Supplemental Material) were analyzed by liquid chromatography-tandem mass spectrometry (LC-MS/MS) with eluent fractions collected at 10-second intervals into 96-well plates containing solid scintillant. Radioactivity in each well was determined using TopCount analysis for the generation of radioactive profiles.

Urine. Predose to 144-hour samples from group 1 and predose to 48-hour samples from group 2 were analyzed using liquid chromatography-mass spectrometry (LC-MS) with eluent fractions collected at 10-second intervals into 96well plates containing solid scintillant. Radioactivity in each well was determined 
using TopCount analysis, and radiochemical profiles were generated based on radioactivity counts. Due to low levels of radioactivity, group 1 samples from 144 hours onward and group 2 samples from 48 hours onward were analyzed by LC-MS/MS only.

Bile. Bile samples from group 2 were pooled by collection interval. Radioactivity in each pooled sample was determined by LSC. Samples were analyzed by LC-MS/MS, and radioactivity was determined as described above.

Feces. Feces from group 1 were pooled to generate one 0 -120-hour pooled sample. Samples from group 2 were pooled to generate one 0-72-hour pool. Radioactivity in each pooled sample was determined by LSC. Reconstituted samples (see Supplemental Material) were analyzed by LC-MS/MS with eluent fractions collected at 10-second intervals into 96-well plates containing solid scintillant. The radioactivity in each well was determined through TopCount analysis, and radioactive profiles were generated based on radioactivity counts.

LC/MS-MS Instrumentation and Conditions. Processed tissue matrices samples were injected with a Shimadzu Nexera SIL-30ACMP autoinjector $\left(10^{\circ} \mathrm{C}\right)$ equipped with a Prominence CBM-20A controller and Nexera LC-30AD pumps (Shimadzu Scientific Instruments, Columbia, MD) that was coupled to a Phenomenex $3 \times 4 \mathrm{~mm} \mathrm{C18}$ guard column (Phenomenex, Torrance, CA), a Waters Atlantis T3 HPLC column $(4.6 \times 250 \mathrm{~mm}, 5 \mu \mathrm{m}$; Waters Corporation, Milford, MA), and an LEAP Technologies PAL HTC-xt fraction collector (LEAP Technologies Inc., Morrisville, NC). Mobile phase A was $0.1 \%$ formic acid in water, and mobile phase B was acetonitrile. Tandem mass spectrometry (MS/MS) was performed in a Thermo Fisher Scientific Q Exactive mass spectrometer (Thermo Fisher Scientific, Waltham, MA) with a positive/negative heated electrospray interface. Detailed LC-MS/MS conditions are listed in Supplemental Material.

\section{Data Analysis for Mass-Balance Study in Monkey}

Pharmacokinetic and Mass-Balance Analyses. Radioanalysis data tables were generated by Debra, version 6.1.1.87 (LabLogic Systems Ltd., Sheffield, UK). PK parameters for both total radioactivity and plasma concentrations of parent were calculated using Phoenix WinNonlin, version 6.4 or higher (Certara USA, Inc., Princeton, NJ) with a noncompartmental approach.

Metabolite Identification. To describe and quantify peaks, a background of $3 \mathrm{cpm}$ was applied to all chromatograms, and the net radioactivity in each peak was expressed as a percentage of total radioactivity in the chromatogram or sample. Metabolite profiles in plasma were reported as a percentage total sample radioactivity and as concentration (ng equivalents/g). For excreta, the percentage of the administered dose excreted as the component represented by the peak was calculated using the following equation: $\%$ of dose $=\%$ of radioactivity in peak $\times \%$ of dose in sample. The percentage dose and concentration of the peak were corrected for extraction and reconstitution recoveries as applicable. M numbers were assigned to peaks as M1 through M6 to match previously reported metabolites along with additional metabolites found in this study assigned up to M17.

\section{Pharmacokinetics of Unlabeled Maribavir in Intact Monkeys after a Single Intravenous or Oral Administration}

Male cynomolgus monkeys of Chinese origin aged 3-4 years $(n=3)$ were used. On day 1 each animal received a single intravenous dose of maribavir at 5 $\mathrm{mg} / \mathrm{kg}$ in a dose volume of $1 \mathrm{ml} / \mathrm{kg}$ in saline containing $10 \%$ ethanol and $40 \%$ propylene glycol 400 . Blood was collected for plasma at predose and at 0.083 , $0.25,0.5,0.75,1,2,4,8,24,36,48,72$, and 96 hours postdose. On day 15, the same animals received a single oral gavage dose of maribavir at $10 \mathrm{mg} / \mathrm{kg}$ in a dose volume of $5 \mathrm{ml} / \mathrm{kg}$ with the same vehicle as intravenous dosing. The feeding tube was flushed with $2-3 \mathrm{ml}$ of water after gavage dosing. Blood was collected for plasma once predose and at $0.25,0.5,0.75,1,2,4,6,8,24,36,48,72$, and 96 hours postdose. Monkey plasma samples added with the internal standard $\left(\mathrm{d}_{7}\right.$-maribavir) were extracted using protein precipitation with organic solvent. Plasma samples were analyzed by high-performance liquid chromatography equipped with an AB SCIEX API 4000 triple quadrupole mass spectrometer using an electrospray ionization source. Negative ions were monitored in the multiple reaction monitoring mode. Quantitation was determined using a weighted linear regression analysis $\left(1 /\right.$ concentration $\left.^{2}\right)$ of peak area ratios of the maribavir and internal standard. Additional details are in the Supplemental Material. Noncompartmental PK analysis was performed for both the intravenous and p.o. arms with Phoenix WinNonlin version 6.4 or higher. The same noncompartmental analysis was also used to calculate PK parameters for previously published studies with maribavir.

\section{In Vitro Metabolism of ${ }^{14} \mathrm{C}$-maribavir in Hepatocytes}

The in vitro metabolism of maribavir was evaluated in cryopreserved hepatocytes isolated from cynomolgus monkeys or humans. ${ }^{14} \mathrm{C}$-Maribavir $(10 \mu \mathrm{M})$ was incubated in $1 \mathrm{ml}$ at $10^{6}$ cells $/ \mathrm{ml}$ for 4 hours, and metabolite profiles were obtained by analyzing hepatocyte extracts by LC /UV/MS and LC/UV/radioactivity flow detector. Further details are described in the Supplemental Material.

\section{Permeability of Maribavir across Caco-2 Cell Monolayer}

Caco-2 cell monolayers were grown to confluence on collagen-coated, microporous, polycarbonate membranes in 12-well Transwell plates (Corning Life Sciences, Tewksbury, MA). Transepithelial electrical resistance was measured with standard procedures before and after the study (Benson et al., 2013); the average transepithelial electrical resistance was $553 \Omega \cdot \mathrm{cm}^{2}$. Compounds tested were maribavir as well as Lucifer Yellow, atenolol, and propranolol. Cells were dosed on the apical or basolateral side and incubated at $37^{\circ} \mathrm{C}$. At 1 and 2 hours, a 200- $\mu 1$ aliquot was taken from the receiver chamber and replaced with fresh assay buffer. Concentrations of test article were determined by LC-MS in ES+ mode. Further details are described in the Supplemental Material.

\section{Semi-PBPK Model for Disposition of Maribavir in Monkeys}

Based on findings from the mass-balance study in BDC and intact monkeys, a semi-PBPK model was developed to describe the kinetics of maribavir in plasma and that of maribavir and its glucuronides in the gastrointestinal (GI) tract. The model construction, parameter estimation, simulations, and sensitivity analyses were conducted with SimBiology version 5.7 hosted in MATLAB R2017b with Optimization Toolbox version 8.0 (The Mathworks Inc., Natick, MA).

Model Construction. The semi-PBPK model comprised a compartmental absorption and transit module for the GI tract (Yu and Amidon, 1999) and three systemic compartments (peripheral, central, and liver) (Fig. 2; Supplemental Fig. 1; Supplemental Tables 1 and 2). The GI model consists of 13 total luminal compartments: stomach (one compartment), duodenum (one), jejunum (two), ileum (four), and colon (five). Within each luminal segment, maribavir is absorbed with first-order kinetics, with the rate $\left(\mathrm{k}_{\mathrm{a}}\right)$ determined by effective permeability $\left(\mathrm{P}_{\text {eff }}\right)$ specific to each segment (see Supplemental Methods and Supplemental Table 3). Human jejunal $\mathrm{P}_{\text {eff }}$ of maribavir was estimated from its measured Caco-2 apparent permeability, which was calibrated by assay controls (atenolol and propranolol) and log-linear regression of $\mathrm{P}_{\text {eff }} \mathrm{P}_{\text {app }}$ (apparent permeability) data (see Supplemental Fig. 2; Supplemental Methods; Supplemental Table 4). Monkey jejunal $\mathrm{P}_{\text {eff }}$ was assumed to be the same as human. The observed plasma concentrations were assumed to resemble that of the central compartment. The liver compartment was necessary to model the first-pass metabolism as well as the metabolic and biliary elimination of maribavir as glucuronides or others. Luminal conversion of maribavir glucuronides to maribavir was assumed to occur in the distal small intestine and throughout the colon, and it was also assumed that the entirety of regenerated maribavir is available for reabsorption. All system parameters, such as intestinal transit times, intestinal radii, and hepatic blood flow, were obtained from literature; drug-specific parameter values were determined in in vitro studies, measured from excreta contents of the BDC group, or estimated from the plasma concentration time course of the BDC group (Supplemental Table 5; Table 1). Further details are described in the Supplemental Material.

Parameter Estimation. Four systemic PK parameters [drug clearance from the liver compartment (Drug_Liver_CL), central-to-peripheral transfer clearance (Drug_Q12), and volumes of the central and peripheral compartments (Drug_Vc_Ref and Drug_Vp_Ref)] were estimated by fitting the BDC group plasma concentrations of maribavir to the central compartment concentration using the constrained nonlinear least-squares method. The observed plasma concentrations were pooled at each time point (because of BDC animal 2 having fewer available plasma concentrations), and an exponential error model was chosen. Termination tolerance on the estimated coefficients, objective function value, and first-order optimality were all set at $10^{-12}$, and maximum iterations were set at 5000. The performance of fitting was evaluated and confirmed with $\% \mathrm{CV}$ of the estimated values (Table 1) and the observed data versus predictions and residuals versus predictions plots (Supplemental Fig. 3, A and B).

Model Qualification and Simulations. By varying the doses and routes of administration (intravenous bolus or oral), plasma concentration versus time profiles of maribavir in intact animals were simulated using the semiPBPK model for $13 \mathrm{mg} / \mathrm{kg}$ i.v. dosing in mass-balance study; for $5 \mathrm{mg} / \mathrm{kg}$ i.v. and $10 \mathrm{mg} / \mathrm{kg}$ p.o. dosing in the unlabeled PK study; and for 20,60, and 


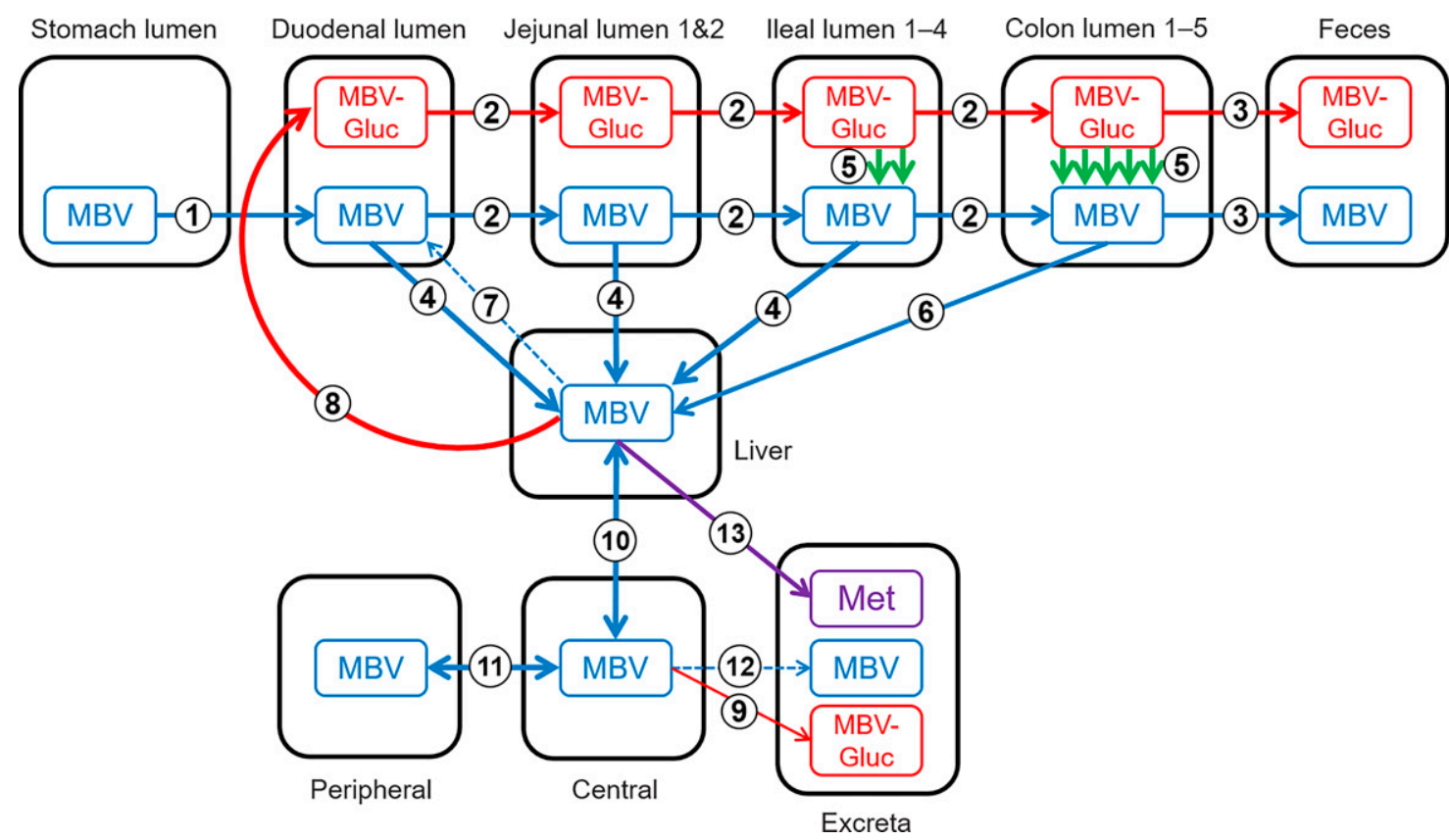

Fig. 2. Simplified diagram for the semi-PBPK model of maribavir disposition in cynomolgus monkeys. Numbers in the graph denote physiologic and biologic processes: (1) transit of substance from stomach to duodenum; (2) transit within the small intestine; (3) transit within the colon and to feces; (4) absorption from the small intestine; (5) conversion of glucuronides to parent, which occurs in the last two compartments of the ileal lumen and throughout the colon; (6) absorption from the colon; (7) biliary excretion of parent; (8) glucuronidation of parent and secretion to duodenal lumen; (9) excretion of glucuronides in urine; (10) transport between the liver and central compartment; (11) transport between the central and peripheral compartments; (12) renal excretion of parent; and (13) all other pathways for parent. The two segments of the jejunum, four segments of the ileum, and five of the colon were not separately depicted in this diagram; the detailed diagram in MATLAB SimBiology is shown in Supplemental Fig. 1. MBV, maribavir (parent); MBV-Gluc, maribavir glucuronides; Met, other metabolites of maribavir.

$180 \mathrm{mg} / \mathrm{kg} /$ day reported in a historical toxicokinetic study (Koszalka et al., 2002). The SUNDIALS solver was used, and absolute tolerance was set at $1.0^{-9}$; dimension analysis was enabled. Observed data were overlaid to and graphically compared with simulated results. Quantitative comparisons included area under the curve (AUC) (intravenous and p.o.), $C_{\max }$, time to peak concentration $\left(T_{\max }\right)$, and bioavailability (p.o. only) as well as the median percentage predictive error (\%PE, defined by eq. 1$)$ :

$$
\% P E=\frac{C_{\text {Pred }}-\bar{C}_{\text {Obs }}}{\bar{C} \text { Obs }} \times 100 \%
$$

in which $\overline{\mathrm{C}}_{\mathrm{Obs}}$ is the mean observed plasma concentration at each time point, and $C_{\text {Pred }}$ is the simulated concentration at corresponding time points. A median $\% \mathrm{PE}$ between $-50 \%$ and $100 \%$, which corresponds to the predicted value being within the 2-fold range of the mean observed value, generally indicates a PBPK model with good predictability (Khalil and Laer, 2014; Zhou et al., 2016; Matsumoto et al., 2019).

Sensitivity Analysis. Sensitivity analyses of the plasma PK time profile of maribavir after a single $10-\mathrm{mg} / \mathrm{kg}$ p.o. administration with respect to parameters in Table 1 were conducted within SimBiology by varying each designated parameter within a range around the final model value. Four drug- and systemspecific parameters [i.e., $P_{\text {eff }}$ in the jejunum, clearance from the liver compartment (Drug_Liver_CL), fraction metabolized $\left(\mathrm{f}_{\mathrm{m}}\right)$ by the direct glucuronidation pathway $\left(\mathrm{f}_{\mathrm{m}}(\mathrm{Gluc})\right)$, and transit rate within the colonic lumen (k_T_Colon)] were found to confer the most impact on the exposure of maribavir.

\section{Results}

Metabolic Pathway of ${ }^{14} \mathrm{C}$-Maribavir after a Single Intravenous Bolus Injection to Cynomolgus Monkeys

After a single $13-\mathrm{mg} / \mathrm{kg}$ i.v. dose of ${ }^{14} \mathrm{C}$-maribavir to male intact monkeys (group 1), radioactivity in plasma declined after the first time point (5 minutes) and was below the limit of quantitation (BLQ) after 72 hours (Fig. 3A; Supplemental Fig. 4). Mean clearance and terminal half-life $\left(t_{1 / 2}\right)$ for radioactivity in plasma were $1700 \mathrm{~g} / \mathrm{h}$ and 19.7 hours, respectively (Table 2). In BDC monkeys (group 2), after a single $13-\mathrm{mg} / \mathrm{kg}$ i.v. dose of ${ }^{14} \mathrm{C}$-maribavir, radioactivity in plasma declined more rapidly than in group 1 and was BLQ by 24 hours postdose (Fig. 3A; Supplemental Fig. 4). In group 2, the clearance of radioactivity in plasma at $3010 \mathrm{~g} / \mathrm{h}$ was $77 \%$ higher than in group 1 ; mean $t_{1 / 2}$ (2.71 hours) was also much shorter than in group 1 (Table 2).

The major route of elimination of radioactivity in intact monkeys (group 1) was via feces, with a mean of $75.2 \%$ excreted over 336 hours (Fig. 4A). The mean percentage of radioactivity recovered in urine was $14.1 \%$ over 336 hours, with the majority of the radioactivity being excreted by 96 hours. The major route of elimination of radioactivity in BDC monkeys (group 2) was via bile, with a mean of $84.0 \%$ excreted over 168 hours postdose (Fig. 4B). The mean percentages of the administered radioactivity recovered in urine and feces from BDC monkeys were $5.16 \%$ and $2.36 \%$, respectively, over 168 hours. The majority of the radioactivity was excreted by 8 hours postdose; radioactivity levels in bile were BLQ by 72 hours. The mean combined radioactivity in all nonbiologic samples was less than $5 \%$ of dose for both groups. The mean overall recovery of the dose administered was $95.3 \%$ and $97.0 \%$ in groups 1 and 2, respectively.

\section{Pharmacokinetics of ${ }^{14} \mathrm{C}$-Maribavir in Plasma after Intravenous Bolus Injection to Monkeys}

The PK profiles of maribavir in plasma after a single intravenous bolus administration in monkeys are depicted in Fig. 3B (individually plotted in Supplemental Fig. 4), and the PK properties are listed in Table 3. The mean clearance in intact animals $(2.99 \mathrm{l} / \mathrm{h})$ was about half of that in BDC animals $(5.72 \mathrm{l} / \mathrm{h})$. Intact animals also displayed a much more pronounced terminal phase (mean $t_{1 / 2}$ of 12.5 hours) than their BDC counterparts $\left(t_{1 / 2} 2.5\right.$ hours). These results were consistent with the aforementioned observations on total radioactivity (Fig. 3A; Supplemental Fig. 4; Table 2). 
TABLE 1

Key system parameters and drug-specific parameters used in construction of the customized semi-physiologically based pharmacokinetic model for maribavir in cynomolgus monkeys

For a full list of parameters, refer to Supplemental Table 5. "Drug" refers to maribavir.

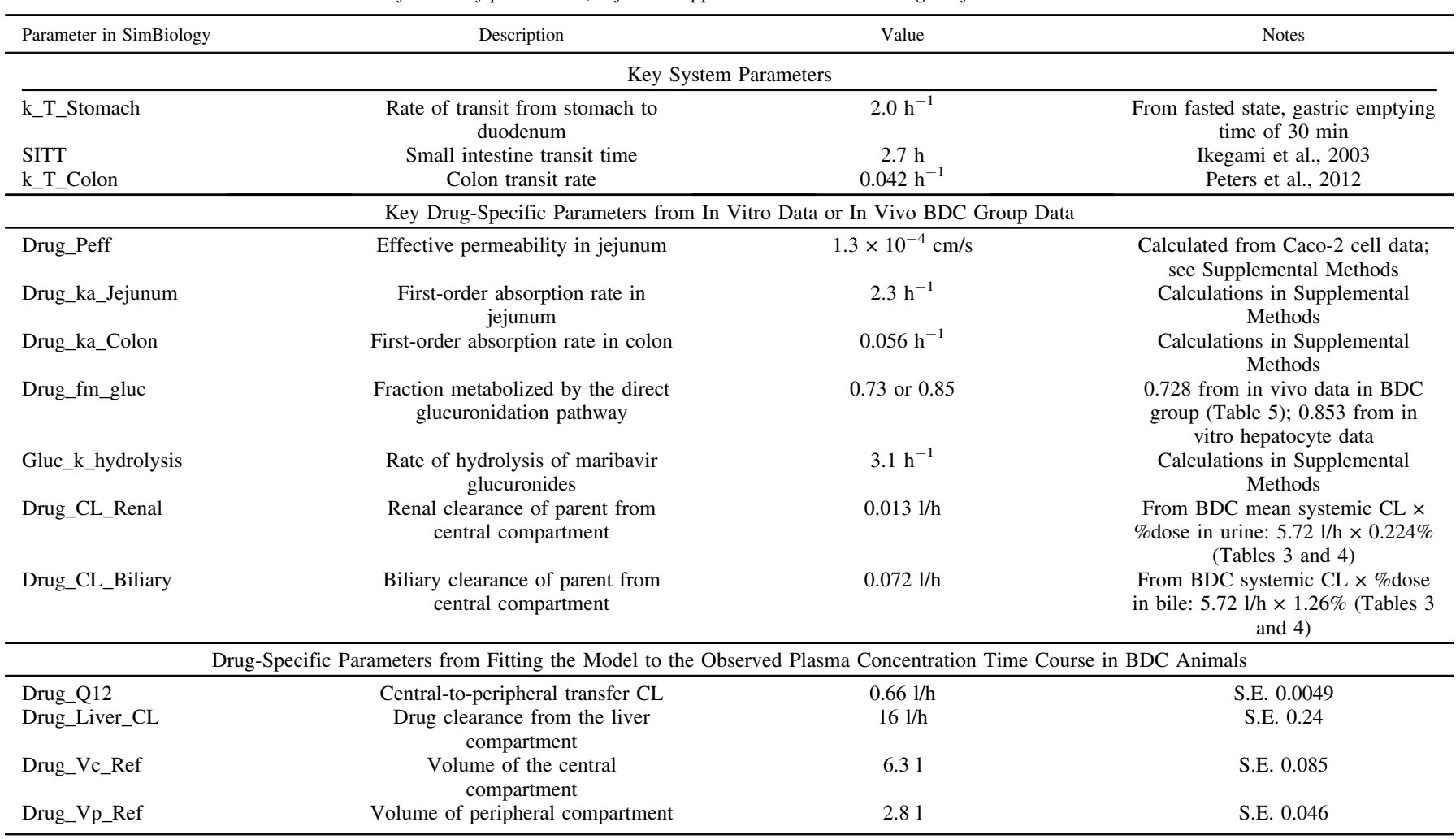

CL, clearance.

\section{Identification and Quantitation of Metabolites of ${ }^{14} \mathrm{C}$-Maribavir in Monkey Plasma and Excreta}

Eleven metabolites of maribavir (Table 4; structures shown in Supplemental Table 6; MS/MS spectra and structural assignment for each fragment ion detailed in Supplemental Figs. 5-17) were tentatively identified from the collected biologic matrices (reconstructed ion chromatograms and radiochromatograms detailed in Supplemental Figs. 18-27). A comprehensive illustration of metabolic pathways of maribavir in monkeys is depicted in Fig. 5. For plasma from both intact and BDC animals, parent was the predominant species associated with radioactivity in circulation at almost all time points, accounting for more than $80 \%$ of all radioactivity in plasma (Supplemental Table 7). Much lower concentrations of the $N$-dealkylated metabolite M4 [also known as VP44469 (Goldwater et al., 2008)], two glucuronides of M4 (M7 and M17) and two direct glucuronides of parent (M11 and M12) were also detected at some time points in plasma, but the combined percentages of these metabolites generally amounted to less than $15 \%$ of circulating radioactivity. At all time points, no individual metabolite accounted for more than $8 \%$ of circulating radioactivity in plasma.

A pronounced difference in the composition of metabolites in excreta was observed between intact and BDC animals. In the excreta from intact animals (group 1), the bulk of radioactivity (58\%-60\% of the administered dose) was recovered as the unchanged parent compound in feces. The dealkylation product M4 generated from cytochrome P450-mediated pathways (Koszalka et al., 2002; Goldwater et al., 2008) represented approximately $9 \%$ of the total dose in pooled feces; in pooled urine, M4 and its glucuronides in total also comprised up to $9 \%$ of administered dose (Table 4). Metabolites M11 and M12, direct glucuronides of maribavir, were present in the urine from intact animals at a combined 3\% total dose. Neither maribavir glucuronides nor M4 glucuronides were detected in the feces of intact animals. In contrast, in BDC animals, the majority (73\%) of administered dose was recovered as M11 and M12 in bile. Biliary secretion of the parent compound itself only accounted for a small fraction $(1.3 \%)$ of the dose; the amount of parent in pooled feces of BDC animals was also small ( $1.5 \%$ total dose). In the urine of BDC monkeys, $0.22 \%$ of the dose was identified as parent, and M4 and its glucuronides combined were $3.6 \%$ of administered dose; thus, both values were numerically lower than the respective $0.89 \%$ and $>7.1 \%$ observed in intact animals (Table 4). M15, a dechlorinated and cysteine-conjugated metabolite, was unique to BDC animals and detected only in bile samples, but it cumulatively represented only $1.4 \%$ of the total dose. Two other metabolites were also detected at low amounts: metabolite M5, with oxidation on $N$-isopropyl, at $<0.43 \%$ of the total dose, and M2, the deribosylated and glucuronidated metabolite, at $<2.5 \%$ of the total dose in both groups (Table 4 ).

\section{In Vitro Metabolism of Maribavir in Cryopreserved Hepatocytes}

After a 4-hour incubation with cynomolgus monkey hepatocytes, ${ }^{14} \mathrm{C}$-maribavir was almost completely metabolized. Major metabolites included M11 and M12 (direct glucuronides) as well as the multiple glucuronides to M4 (N-dealkylation). M11 and M12 combined and M4 glucuronides represented $85.3 \%$ and $10.4 \%$ of total radioactive peak areas, respectively. After a 4-hour incubation with human hepatocytes, parent, M4, M4-glucuronides, and one of the direct glucuronides to parent (M11 or M12) represented $47.2 \%, 31.1 \%, 10.2 \%$, and $10 \%$ of total radioactive peak areas, respectively; M4 plus its glucuronides 

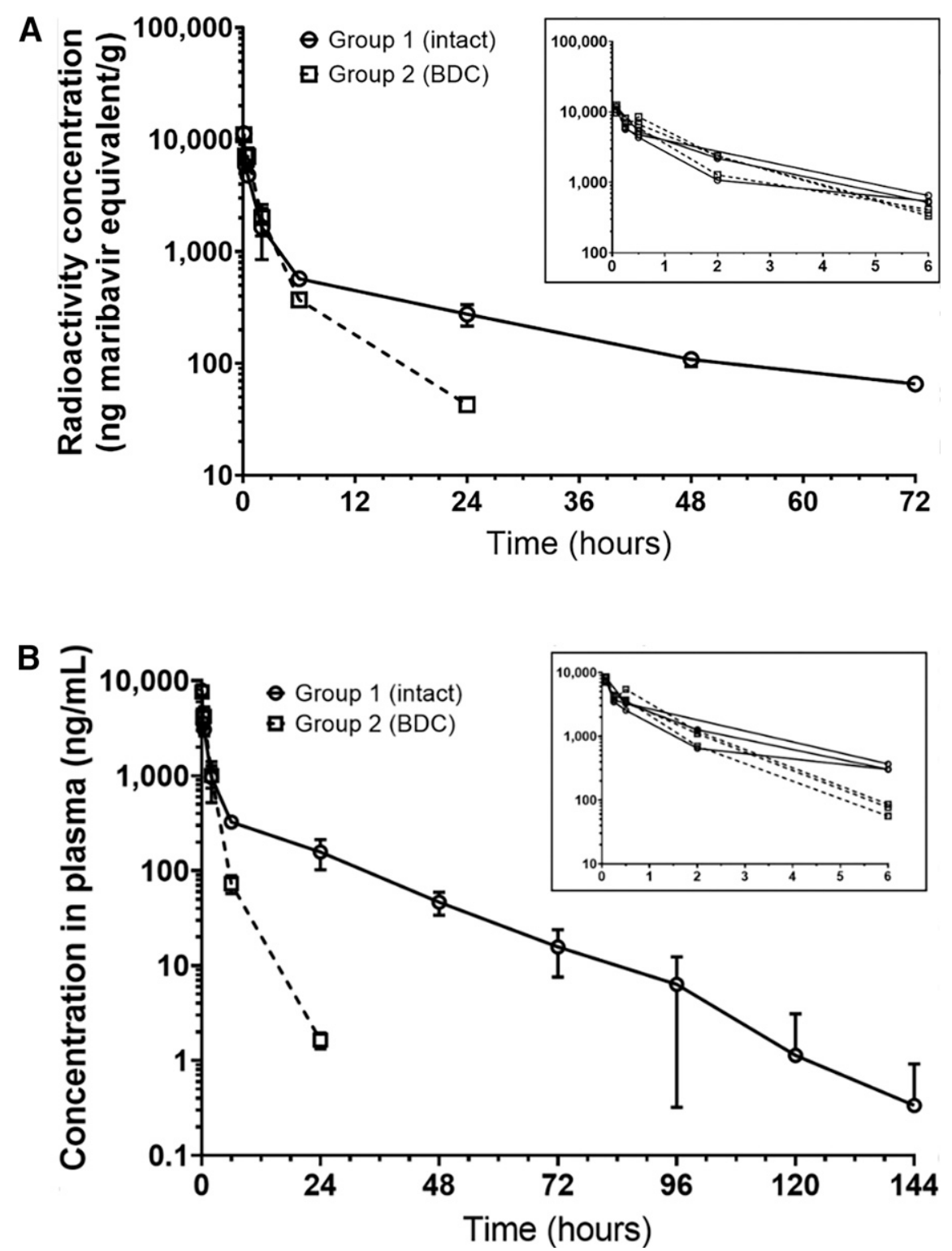

Fig. 3. (A) Total radioactivity in plasma vs. time profile after a single intravenous bolus administration of $13 \mathrm{mg} / \mathrm{kg}{ }^{14} \mathrm{C}$-maribavir to intact animals (group 1 , circles) or BDC animals (group 2, squares) as determined by LSC. (B) Concentration of maribavir in plasma vs. time profile after a single intravenous bolus administration of $13 \mathrm{mg} / \mathrm{kg}{ }^{14} \mathrm{C}$-maribavir to intact animals (group 1, circles) or BDC animals (group 2, squares) as determined by LC-MS/MS. Insets show a zoomed-in view of the time course from zero to 6 hours postdose. Missing samples are not plotted, and BLQ data are treated as zeros. $N=3$ for both groups, error bars denote standard deviation, and $N=2$ for group 2's 24-hour sample in panel (B).

and M11/M12 represented $78 \%$ and $19 \%$ of metabolism of maribavir in human hepatocytes. Other metabolites were present at trace amounts (each $<1 \%$ ) for both monkey and human hepatocyte incubations.
Permeability of Maribavir Across Cultured Caco-2 Cell Monolayer

The apparent permeability for maribavir in the apical-to-basolateral and basolateral-to-apical directions were $5.9 \times 10^{-6} \mathrm{~cm} / \mathrm{s}$ and $33.7 \times 10^{-6} \mathrm{~cm} / \mathrm{s}$, respectively. The apical-to-basolateral apparent

TABLE 2

Noncompartmental PK parameters for radioactivity in plasma collected from male cynomolgus monkeys after a single intravenous administration of ${ }^{14} \mathrm{C}$-maribavir (both groups at $13 \mathrm{mg} / \mathrm{kg})$. Group 1: intact monkeys $(n=3)$, group 2: BDC monkeys $(n=3)$

\begin{tabular}{lcc}
\hline PK Parameter on Total Radioactivity in Plasma & Group 1 (Intact), & Group 2 (BDC), \\
Mean \pm S.D.
\end{tabular}

$\mathrm{AUC}_{0-t}$, area under the conc.-time curve from time 0 to the last measurable time point; $\mathrm{AUC}_{0-\infty}$, area under the conc.-time curve from zero to infinity; $\mathrm{CL}$, clearance; $V_{\mathrm{ss}}$, volume of distribution at steady state. 
A Cumulative recovery of radioactivity: Group 1

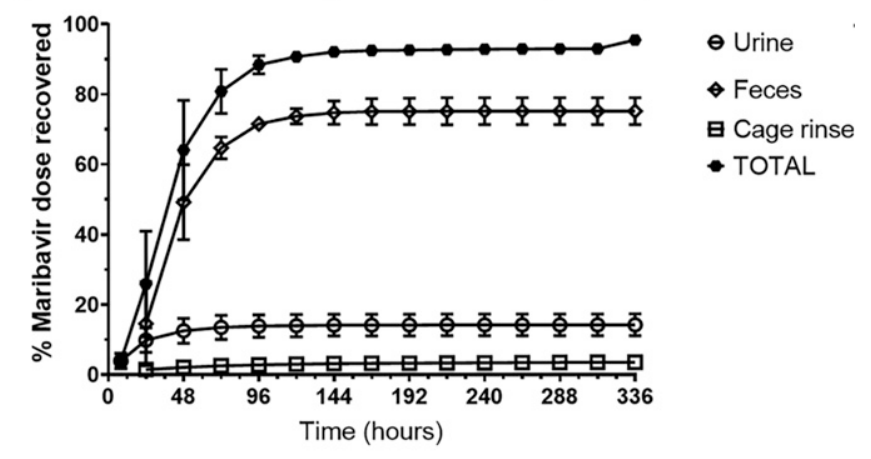

B Cumulative recovery of radioactivity: Group 2

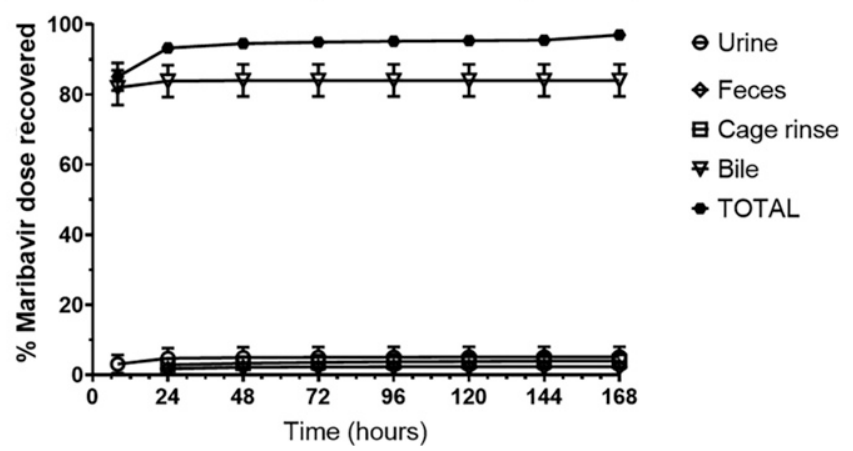

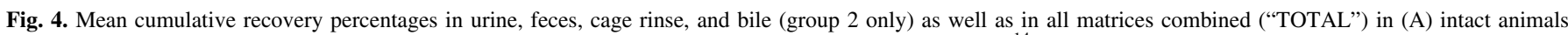

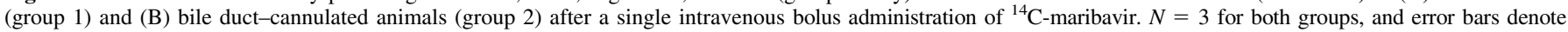
standard deviation.

permeability for Lucifer Yellow, atenolol, and propranolol were $0.26 \times 10^{-6} \mathrm{~cm} / \mathrm{s}, 0.36 \times 10^{-6} \mathrm{~cm} / \mathrm{s}$, and $17.1 \times 10^{-6} \mathrm{~cm} / \mathrm{s}$, respectively.

\section{Semi-PBPK Model for EHR of Maribavir in Monkeys}

Parameter Estimations. The final PK parameters derived from fitting the semi-PBPK model to the observed plasma concentration over time from BDC monkeys (group 2) are listed in Table 1 (see also Supplemental Results and Discussion). The fitted concentration and observed data over time are shown in Fig. 6A; observed versus predicted and residual versus prediction graphs are in Supplemental Fig. 3, A and B. The overall residual error of the fitting was 0.256 .

\section{TABLE 3}

Noncompartmental PK parameters for conc. of maribavir in plasma from male cynomolgus monkeys after a single intravenous bolus administration of ${ }^{14} \mathrm{C}$-maribavir [groups $1(n=3)$ and $2(n=3), 13 \mathrm{mg} / \mathrm{kg}$ ].

\begin{tabular}{lcc}
\hline $\begin{array}{l}\text { PK Parameter on Maribavir } \\
\text { Concentration in Plasma }\end{array}$ & $\begin{array}{c}\text { Group 1 (Intact), } \\
\text { Mean } \pm \text { S.D. }\end{array}$ & $\begin{array}{c}\text { Group 2 (BDC), } \\
\text { Mean } \pm \text { S.D. }\end{array}$ \\
\hline $\mathrm{AUC}_{0-\mathrm{t}}(\mathrm{h} \cdot \mathrm{mg} / \mathrm{l})$ & $17.4 \pm 3.9$ & $9.33 \pm 1.7$ \\
$\mathrm{AUC}_{0-\infty}(\mathrm{h} \cdot \mathrm{mg} / \mathrm{l})$ & $17.4 \pm 3.9$ & $9.36 \pm 1.6$ \\
$\mathrm{C}_{0}(\mathrm{mg} / \mathrm{l})$ & $9.96^{a}$ & $10.4 \pm 1.3$ \\
$t_{1 / 2}(\mathrm{~h})$ & $12.5 \pm 1.1$ & $2.49 \pm 1.2$ \\
$\mathrm{CL}(\mathrm{l} / \mathrm{h})$ & $2.99 \pm 0.73$ & $5.72 \pm 0.90$ \\
$V_{\mathrm{ss}}(\mathrm{l})$ & $35.4 \pm 6.5$ & $7.09 \pm 1.5$ \\
\hline
\end{tabular}

$\mathrm{AUC}_{0-\mathrm{t}}$, area under the conc.-time curve from time 0 to the last measurable time point; $\mathrm{AUC}_{0-\infty}$, area under the conc.-time curve from zero to infinity; $\mathrm{C}_{0}$, initial conc.; $\mathrm{CL}$, clear ance; $V_{\mathrm{ss}}$, volume of distribution at steady state.

${ }^{a}$ For $\mathrm{C}_{0}$, only two animals in group 1 were involved in calculating the mean because of one animal missing the 5-minute PK time point.
Simulations for Intravenous Bolus Dosing of Maribavir in Monkeys. Two $f_{m}($ Gluc) values were used in simulations: 0.853 from the in vitro hepatocyte data or 0.728 from the metabolite content in the pooled bile of BDC monkeys. Graphic results for simulated and observed plasma concentrations of maribavir in intact monkeys administered with ${ }^{14} \mathrm{C}$-maribavir (13 $\mathrm{mg} / \mathrm{kg}$ mass equivalent) or $5 \mathrm{mg} / \mathrm{kg}$ unlabeled maribavir are shown in Fig. 6B and derived PK properties in Table 5. At both doses, the simulations were able to predict the initial phase of rapid decline ( 0 to $4-6$ hours postdose) as well as the prolonged terminal phase (after 4-6 hours postdose) in maribavir plasma concentrations. The observed intergroup difference in predictability of the time course could potentially be explained by the contribution of glucuronidation in the overall clearance: Simulation with $\mathrm{f}_{\mathrm{m}}(\mathrm{Gluc})=0.853$ and 0.728 seemingly presented a better match for terminal phase and AUC for the $13-\mathrm{mg} / \mathrm{kg}$ i.v. group and the $5 \mathrm{mg} / \mathrm{kg}$ i.v. group, respectively. The median $\%$ PE for $13 \mathrm{mg} / \mathrm{kg}$ i.v. was $12 \%$ at $\mathrm{f}_{\mathrm{m}}$ (Gluc) $=0.853$ and $-49 \%$ at $\mathrm{f}_{\mathrm{m}}($ Gluc $)=0.728$; median \%PE for $5 \mathrm{mg} / \mathrm{kg}$ i.v. was $81 \%$ at $\mathrm{f}_{\mathrm{m}}$ (Gluc) $=0.853$ and $30 \%$ at $\mathrm{f}_{\mathrm{m}}($ Gluc $)=0.728$. The overall median $\% \mathrm{PE}$ for all available PK data points after intravenous dosing in intact animals was $38 \%$ at $\mathrm{f}_{\mathrm{m}}$ (Gluc) $=0.853$ and $1 \%$ at $\mathrm{f}_{\mathrm{m}}$ (Gluc) $=0.728$. The $\mathrm{f}_{\mathrm{m}}$ (Gluc) of 0.728 was eventually selected for all follow-up simulations because it is a direct in vivo measurement and also produced a slightly better overall $\% \mathrm{PE}$ and predictive profile across the concentration range (Supplemental Fig. 28).

Simulations for Oral Dosing of Maribavir and Qualification of the Semi-PBPK Model in Monkeys. The semi-PBPK model with $\mathrm{f}_{\mathrm{m}}$ (Gluc) $=0.728$ was able to capture the plasma concentration profiles after a single $10-\mathrm{mg} / \mathrm{kg}$ p.o. dose to intact monkeys (Fig. 6C) during both the apparent absorption and terminal phases; median \% PE was 38\%. Derived PK parameters, such as AUC, 
TABLE 4

Descriptions and cumulative percentages of dose recovered in each pooled biologic matrix for maribavir and its metabolites after a single intravenous bolus administration to intact (group 1) or BDC (group 2) cynomolgus monkeys.

\begin{tabular}{|c|c|c|c|c|c|c|}
\hline \multirow[b]{2}{*}{ Metabolite } & \multirow[b]{2}{*}{ Description } & \multicolumn{2}{|c|}{ Percentage of Dose Administered to Group 1} & \multicolumn{3}{|c|}{ Percentage of Dose Administered to Group 2} \\
\hline & & $\begin{array}{c}\text { Urine } \\
(0-144 \mathrm{~h})\end{array}$ & $\begin{array}{c}\text { Feces } \\
(0-168 \mathrm{~h})^{a}\end{array}$ & $\begin{array}{c}\text { Urine } \\
(0-48 \mathrm{~h})\end{array}$ & $\begin{array}{c}\text { Feces } \\
(0-48 \mathrm{~h})\end{array}$ & $\begin{array}{c}\text { Bile } \\
(0-24 \mathrm{~h})\end{array}$ \\
\hline M15 & Loss of chlorine + cysteine conjugate & ND & ND & ND & ND & 1.42 \\
\hline M1 & Glucuronide conjugate to M4 & 2.16 & ND & 1.19 & ND & ND \\
\hline M7 & Glucuronide conjugate to M4 & 2.62 & ND & 1.14 & ND & 3.77 \\
\hline M16 & Glucuronide conjugate to M4 & 0.297 & ND & ND & ND & 0.992 \\
\hline $\mathrm{M} 17^{\mathrm{b}}$ & Glucuronide conjugate to M4 & $0-1.85$ & ND & $0-0.596$ & ND & $0-1.83$ \\
\hline $\mathrm{M} 2^{\mathrm{b}}$ & Loss of ribose + glucuronide & $0-1.85$ & ND & $0-0.596$ & 0.0282 & $0-1.83$ \\
\hline M10 & Glucuronide conjugate to parent & 0.402 & ND & ND & ND & ND \\
\hline M4 & Loss of propyl & 2.0 & $8.80-9.34$ & 0.678 & 0.511 & ND \\
\hline M5 & Oxidation on propyl & ND & $0.416-0.433$ & 0.0457 & ND & ND \\
\hline M11 & Glucuronide conjugate to parent & 1.2 & ND & 0.334 & ND & 34.2 \\
\hline M12 & Glucuronide conjugate to parent & 2.01 & ND & 0.551 & ND & 38.6 \\
\hline Maribavir & Parent & 0.823 & $57.9-60.3$ & 0.224 & 1.45 & 1.26 \\
\hline
\end{tabular}

ND, not detected or below the limit of quantitation ( $1 \%$ of run and $10 \mathrm{cpm}$ peak height).

${ }^{a}$ The lower dose percentage value was based on pooled fecal samples from all three animals from 0 to 120 hours. Only one animal generated feces from 120 to 168 hours; the upper value reflected the addition of dose percentage from this single animal after 120 hours to the group's pre-120-hour value.

${ }^{b}$ When M17 and M2 are both present in the same matrix, they are indistinguishable in the radio and ion chromatogram as a single peak. Dose percentage values derived from this combined peak were therefore reported.

$C_{\max }$, and bioavailability (F) were also well predicted (Table 5). The fraction absorbed $\left(\mathrm{F}_{\mathrm{a}}\right)$ was estimated at $67 \%$ after a single p.o. dose. For multiple dosing, observed plasma profiles of maribavir after b.i.d. dosing at 10, 30, and $90 \mathrm{mg} / \mathrm{kg}(20,60$, and
$180 \mathrm{mg} / \mathrm{kg} /$ day total) were all reasonably characterized by model predictions (Fig. 6, D and E), and median \%PE was 17\%, $20 \%$, and $29 \%$ for 10,30 , and $90 \mathrm{mg} / \mathrm{kg}$ b.i.d. regimens, respectively.

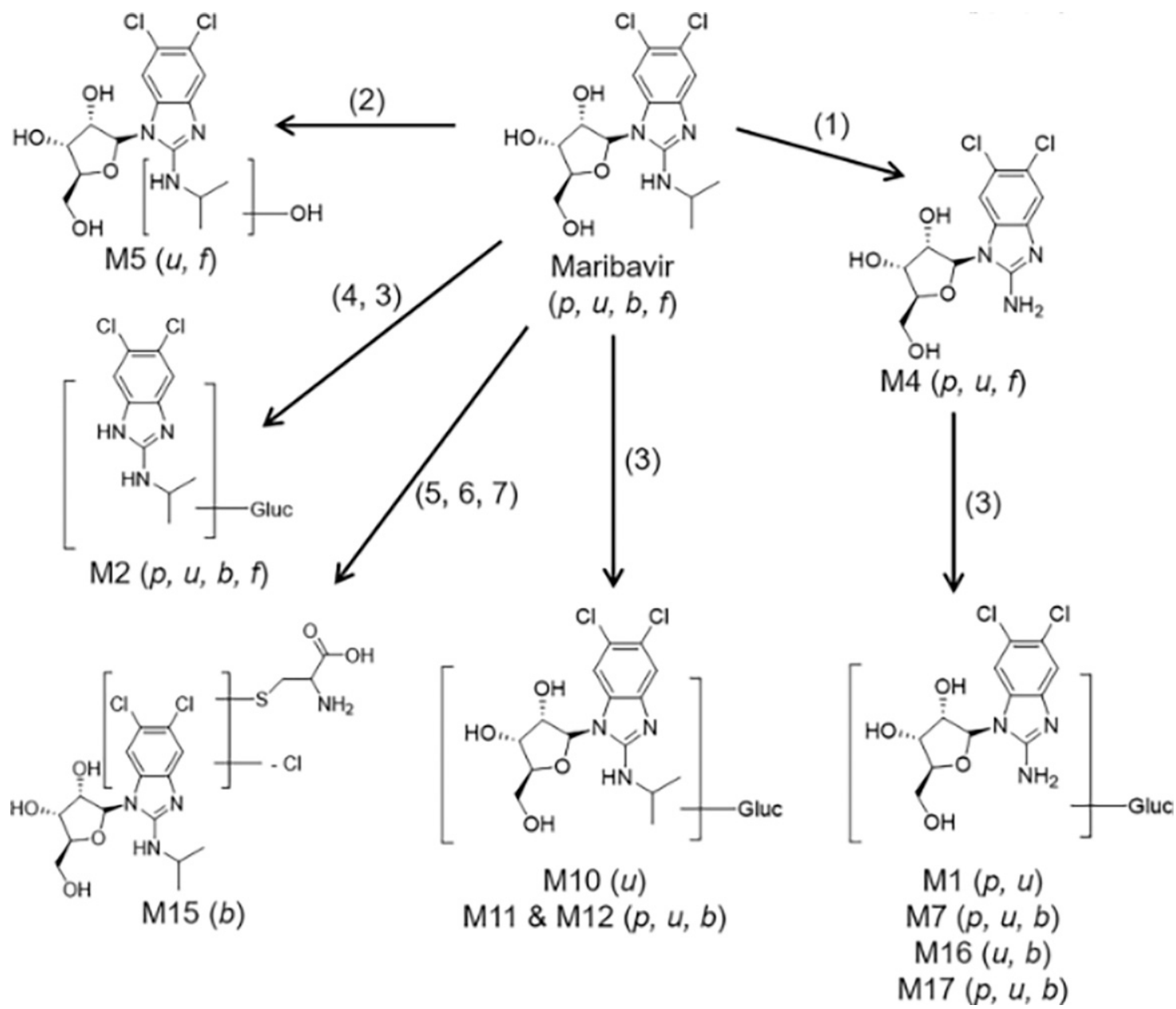

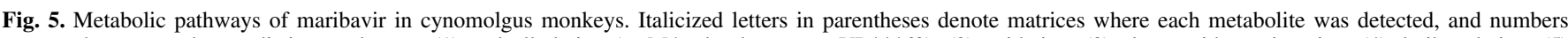

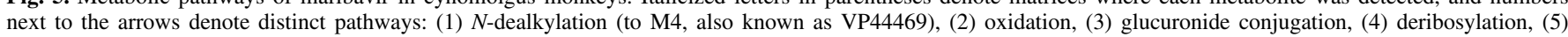
dechlorination, (6) glutathione (GSH) conjugation, and (7) amide hydrolysis after GSH conjugation. $b$, bile; $f$, feces; Gluc, glucuronide; $p$, plasma; $u$, urine. 
A
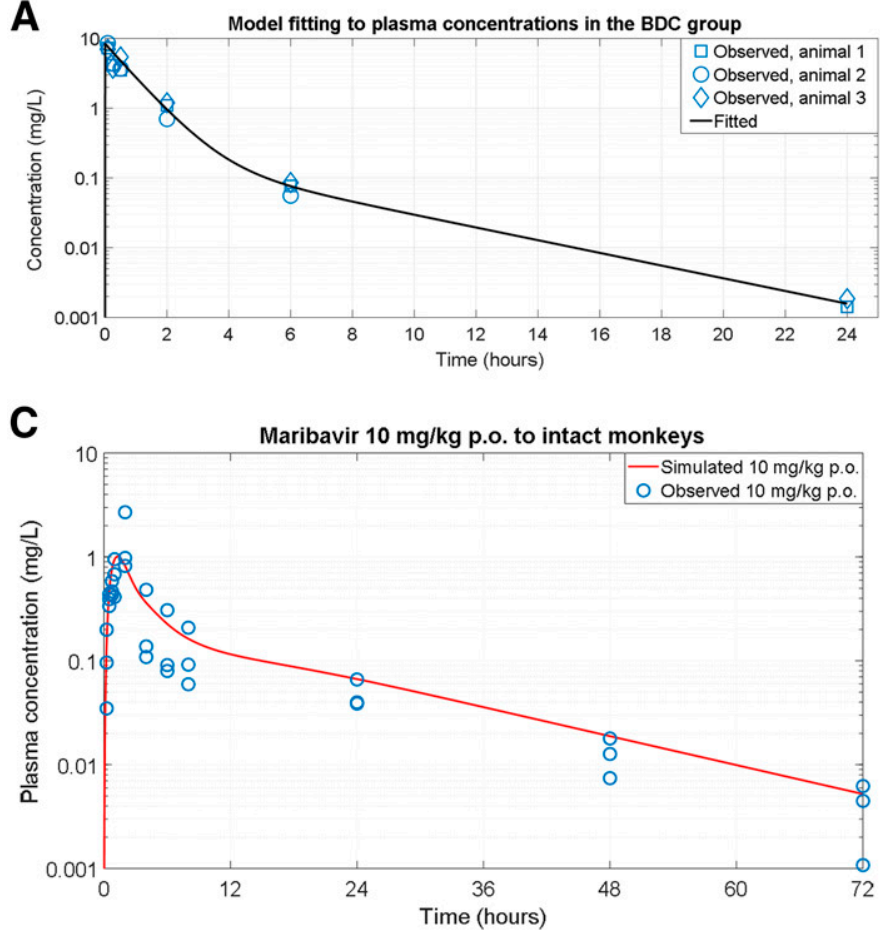

$E$

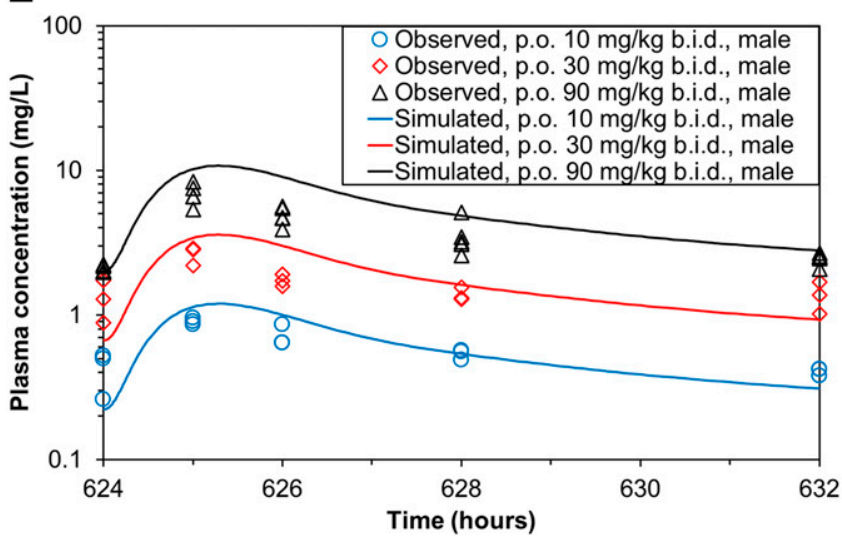

B Maribavir $5 \mathrm{mg} / \mathrm{kg}$ and $13 \mathrm{mg} / \mathrm{kg}$ i.v. bolus in intact monkeys

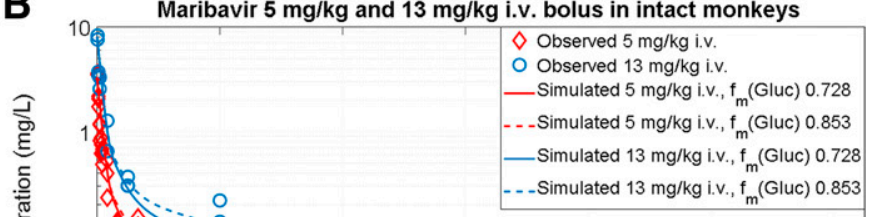

- - Simulated $13 \mathrm{mg} / \mathrm{kg}$ i.v., $f_{\text {(Gluc) }} 0.853$

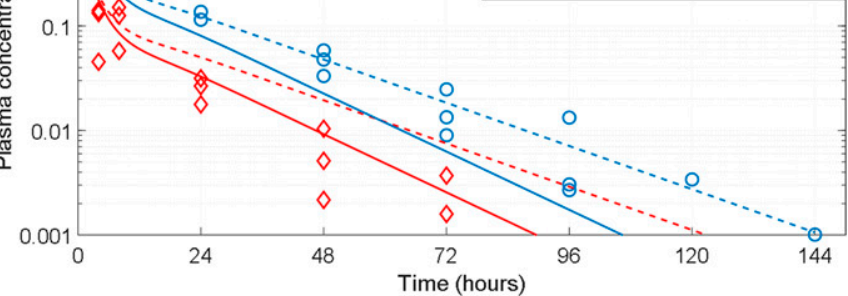

D

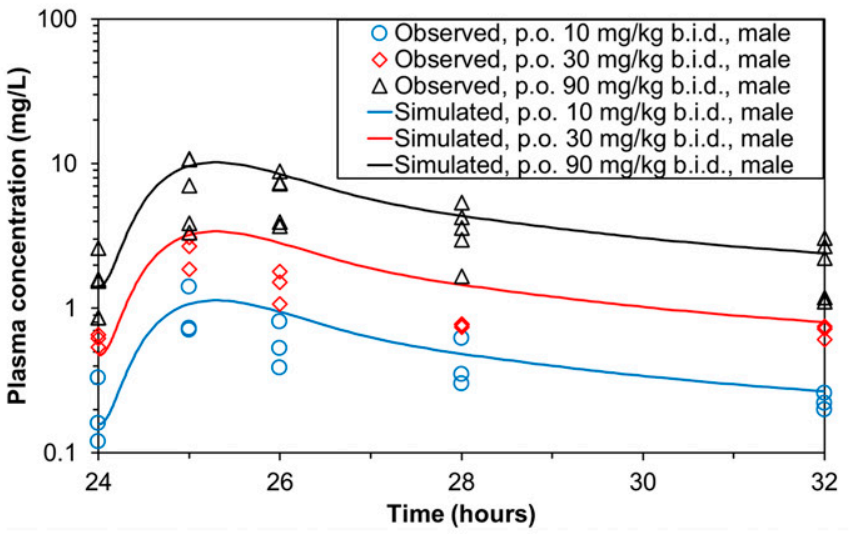

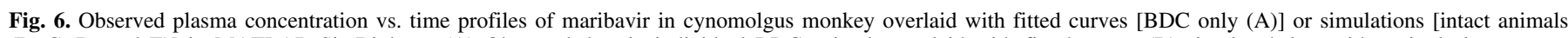

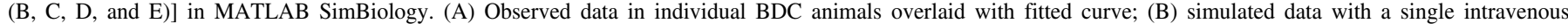

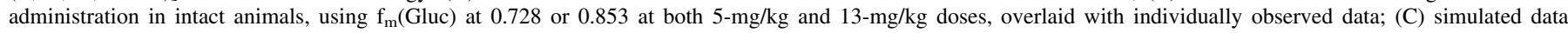

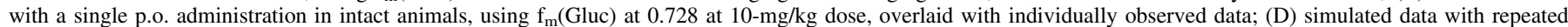

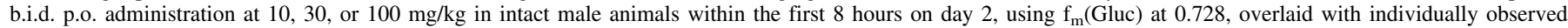

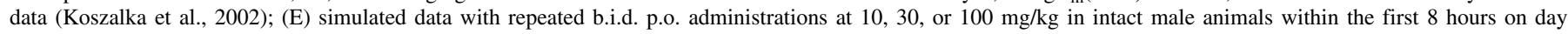
27 , using $\mathrm{f}_{\mathrm{m}}$ (Gluc) at 0.728 , overlaid with individually observed data (Koszalka et al., 2002).

Sensitivity Analyses. Important determinants for systemic exposure of maribavir after a single p.o. dose include the overall $\mathrm{f}_{\mathrm{m}}$ (Gluc) (Fig. 7A), k_T_Colon (Fig. 7B), $\mathrm{P}_{\text {eff }}$ in the small intestine (Supplemental Fig. 29A; Supplemental Results and Discussion), and Drug_Liver_CL (Supplemental Fig. 29B). The AUC in plasma could be increased by increasing $\mathrm{f}_{\mathrm{m}}$ (Gluc), increasing colonic transit time, increasing $\mathrm{P}_{\mathrm{eff}}$, or decreasing Drug_Liver_CL. Notably, a change in Drug_Liver_CL would result in little change in the terminal slope; the latter is largely determined by $f_{m}$ (Gluc), $k_{-} T \_C o l o n$, and, to a lesser degree, $P_{\text {eff }}$. This phenomenon demonstrates the prominent effects of the EHR on systemic exposure of maribavir in monkeys. Systemic $C_{\max }$, on the other hand, is largely driven by $\mathrm{P}_{\text {eff }}$ and Drug_Liver_CL but not by the other two major parameters. Variations in other parameters, such as small intestine transit time (SITT), rate of hydrolysis of maribavir glucuronides (Gluc_k_hydrolysis), and intercompartmental drug clearance
(Drug_Q12), also lead to changes in plasma exposures of maribavir, but their effects are comparatively minor (Supplemental Fig. 29, C-E).

\section{Discussion}

We thoroughly investigated the biotransformation and clearance pathways of maribavir in cynomolgus monkeys by utilizing intact and bile duct-cannulated models and in vitro approaches. We demonstrated that in monkeys, maribavir is primarily metabolized by direct glucuronidation and that these glucuronides, after biliary secretion, can be efficiently converted to maribavir and reabsorbed into circulation. Consequently, in intact animals, maribavir present in gut lumen after intravenous dosing resulted in a higher apparent volume of distribution (Vd) than in BDC animals (Table 2). Conversely, in the BDC group, both the faster clearance due to lack of recirculation and a smaller Vd led to a much shorter 
TABLE 5

PK properties observed in in vivo studies with maribavir in cynomolgus monkeys and estimated from the semi-PBPK model. The $\mathrm{f}_{\mathrm{m}}(\mathrm{Gluc})$ was set as 0.728 unless specified otherwise. The twice-daily doses were administered 8 hours apart within each dosing day

\begin{tabular}{|c|c|c|}
\hline PK Properties on Maribavir in Plasma & Observed, Mean \pm S.D. & Estimated/Predicted by Semi-PBPK Model \\
\hline \multicolumn{3}{|c|}{$13 \mathrm{mg} / \mathrm{kg}{ }^{14} \mathrm{C}$-Maribavir Intravenous Bolus, Single Dose } \\
\hline $\mathrm{AUC}_{0-\mathrm{t}}(\mathrm{mg} \cdot \mathrm{h} / \mathrm{l})$ & $17.4 \pm 3.9$ & $\begin{array}{l}12.5\left(\mathrm{f}_{\mathrm{m}}(\text { Gluc })=0.728\right) \\
15.1\left(\mathrm{f}_{\mathrm{m}}(\text { Gluc })=0.853\right)\end{array}$ \\
\hline $\mathrm{CL}(1 / \mathrm{h})$ & $2.99 \pm 0.73$ & $\begin{array}{l}4.02\left(\mathrm{f}_{\mathrm{m}}(\text { Gluc })=0.728\right) \\
\left.3.33\left(\mathrm{f}_{\mathrm{m}} \text { (Gluc }\right)=0.853\right)\end{array}$ \\
\hline $\mathrm{V}_{\mathrm{ss}}(\mathrm{l})$ & $35.4 \pm 6.5$ & $\begin{array}{l}35.2\left(\mathrm{f}_{\mathrm{m}}(\text { Gluc })=0.728\right) \\
45.8\left(\mathrm{f}_{\mathrm{m}}(\text { Gluc })=0.853\right)\end{array}$ \\
\hline \multicolumn{3}{|c|}{$5 \mathrm{mg} / \mathrm{kg}$ Maribavir Intravenous Bolus, Single Dose } \\
\hline $\mathrm{AUC}_{0-\mathrm{t}}(\mathrm{mg} \cdot \mathrm{h} / \mathrm{l})$ & $4.55 \pm 0.71$ & 5.05 \\
\hline $\mathrm{CL}(1 / \mathrm{h})$ & $4.47 \pm 0.18$ & 4.06 \\
\hline $\mathrm{V}_{\mathrm{ss}}(1)$ & $36.8 \pm 14.7$ & 35.4 \\
\hline \multicolumn{3}{|c|}{$10 \mathrm{mg} / \mathrm{kg}$ Maribavir Oral Gavage, Single Dose } \\
\hline $\mathrm{AUC}_{0-\mathrm{t}}(\mathrm{mg} \cdot \mathrm{h} / \mathrm{l})$ & $6.13 \pm 3.6$ & 6.21 \\
\hline$C_{\max }(\mathrm{mg} / \mathrm{l})$ & $1.50 \pm 1.0$ & 1.01 \\
\hline$T_{\max }(\mathrm{h})$ & $2 \pm 0$ & 1.31 \\
\hline & $0.661 \pm 0.33$ & 0.615 \\
\hline $\mathrm{F}_{\mathrm{a}}$ & N/A & 0.671 \\
\hline \multicolumn{3}{|c|}{10 mg/kg Twice-Daily Maribavir Oral Gavage, Multiple Dose } \\
\hline AUC (mg.h/l), day $2,0-8 \mathrm{~h}$ & $3.60 \pm 1.2$ & 4.41 \\
\hline$C_{\max }(\mathrm{mg} / \mathrm{l})$, day $2,0-8 \mathrm{~h}$ & $0.97 \pm 0.39$ & 1.13 \\
\hline AUC $(\mathrm{mg} \cdot \mathrm{h} / \mathrm{l})$, day $27,0-8 \mathrm{~h}$ & $4.60 \pm 0.33$ & 4.83 \\
\hline$C_{\max }(\mathrm{mg} / \mathrm{l})$, day $27,0-8 \mathrm{~h}$ & $0.90 \pm 0.05$ & 1.20 \\
\hline \multicolumn{3}{|c|}{30 mg/kg Twice-Daily Maribavir Oral Gavage, Multiple Dose } \\
\hline AUC $(\mathrm{mg} \cdot \mathrm{h} / \mathrm{l})$, day $2,0-8 \mathrm{~h}$ & $8.70 \pm 1.2$ & 13.2 \\
\hline$C_{\max }(\mathrm{mg} / \mathrm{l})$, day $2,0-8 \mathrm{~h}$ & $2.55 \pm 0.63$ & 3.40 \\
\hline AUC $(\mathrm{mg} \cdot \mathrm{h} / \mathrm{l})$, day $27,0-8 \mathrm{~h}$ & $12.8 \pm 1.4$ & 14.5 \\
\hline$C_{\max }(\mathrm{mg} / \mathrm{l})$, day $27,0-8 \mathrm{~h}$ & $2.63 \pm 0.38$ & 3.59 \\
\hline \multicolumn{3}{|c|}{90 mg/kg Twice-Daily Maribavir Oral Gavage, Multiple Dose } \\
\hline AUC $(\mathrm{mg} \cdot \mathrm{h} / \mathrm{l})$, day $2,0-8 \mathrm{~h}$ & $32.1 \pm 11$ & 39.7 \\
\hline$C_{\max }(\mathrm{mg} / \mathrm{l})$, day $2,0-8 \mathrm{~h}$ & $7.3 \pm 3.5$ & 10.2 \\
\hline AUC $(\mathrm{mg} \cdot \mathrm{h} / \mathrm{l})$, day $27,0-8 \mathrm{~h}$ & $30.6 \pm 3.2$ & 43.5 \\
\hline$C_{\max }(\mathrm{mg} / \mathrm{l})$, day $27,0-8 \mathrm{~h}$ & $6.9 \pm 1.1$ & 10.8 \\
\hline
\end{tabular}

$\mathrm{AUC}_{0-\mathrm{t}}$, area under the conc.-time curve from time 0 to the last measurable time point; $\mathrm{CL}$, clearance; $\mathrm{F}$, bioavailability; $\mathrm{F}_{\mathrm{a}}$, fraction absorbed; N/A, not available; $T_{\text {max }}$, time to peak conc.; $\mathrm{V}_{\mathrm{ss}}$, volume of distribution at steady state.

maribavir $t_{1 / 2}$ (Fig. 3B; Table 2). A semi-PBPK model was then constructed with data from literature in in vitro and in vivo BDC animals (Supplemental Table 5; Table 1) ; this qualified model captured the pharmacokinetics of maribavir in intact monkeys after either intravenous or oral administration and also identified important compound- and animal-related parameters that impact the kinetics of recirculation.

A previous mass-balance study in intact monkeys (Koszalka et al., 2002) found a large amount of radioactivity in feces as parent and concluded that maribavir was secreted into the bile and subsequently reabsorbed. It may be tempting to claim that the biliary secretion was facilitated through efflux of parent compound at the canalicular surface of hepatocytes (Patel et al., 2016). In this study, we clarified that biliary section of parent drug was actually a minor pathway despite maribavir being a substrate of efflux transporters (Welty et al., 2018; Song et al., 2019a) and that the biliary secretion is primarily facilitated through various glucuronides of maribavir. We assumed that the liver is the primary organ in which glucuronidation of maribavir occurs because of the low percentage of total (\%dose) recovered in urine as maribavir glucuronides (Table 4) and because maribavir is an in vitro substrate of human UDP-glucuronosyltransferase (UGT) 1A1, UGT1A3, and UGT2B7 (unpublished data). Additionally, in monkeys the liver expresses the highest levels of UGT1A and UGT2B and displays much stronger glucuronidation activities than do other organs (Albert et al., 2000).

The hydrolysis of glucuronides by the gut microbiome is mediated by $\beta$-glucuronidases (GUSs) (Zenser et al., 1999). Bacterial GUSs are known to metabolize glucuronides, which occurs in the lumen of distal ileum and colon (Zenser et al., 1999; Koppel et al., 2017; Tropini et al., 2017). The degradation rate constant of typical $O$-glucuronides by colonic microbiota was estimated at 3.1 hour $^{-1}$ in monkeys [derived from (Kim and Jin, 2001; Peters, 2012; Sender et al., 2016); see Supplemental Methods]. Even considering uncertainty related to different $O$-glucuronide substrates, the GUS-mediated hydrolysis of glucuronides is still significantly faster than the typical colon transit rate in monkeys $\left(0.042\right.$ hour $\left.^{-1}\right)$. After biliary secretion likely mediated by transporters (Patel et al., 2016), maribavir glucuronides travel along the GI lumen to the distal ileum and colon, where GUS-expressing bacteria reside. Indeed, between intact and BDC groups, maribavir plasma concentration time courses diverged between 2 and 6 hours postintravenous dose (Fig. 3B), matching SITT around 2.7 hours in monkeys (Table 1) (Ikegami et al., 2003). It is of note that the "second peak" of plasma concentration commonly observed for other enterohepatically recirculated compounds was not apparent in the mass-balance study (Fig. 3) but was evident in another study (Fig. $6 \mathrm{~B} ; 5 \mathrm{mg} / \mathrm{kg}$ group) at $\sim 8$ hours postdose; this may be attributed to different blood collection schedules of the two studies.

Given the abundant knowledge of GI tract physiology and its successful usage for modeling drug absorption (Yu and Amidon, 1999), we created a customized semi-PBPK model to integrate in vivo and in vitro data for maribavir and its metabolites in monkeys. A previous PBPK model (Wu, 2012) provided a theoretical basis on the impact of glucuronide bioconversion to $\mathrm{PK}$ of the aglycone, especially on the latter's 

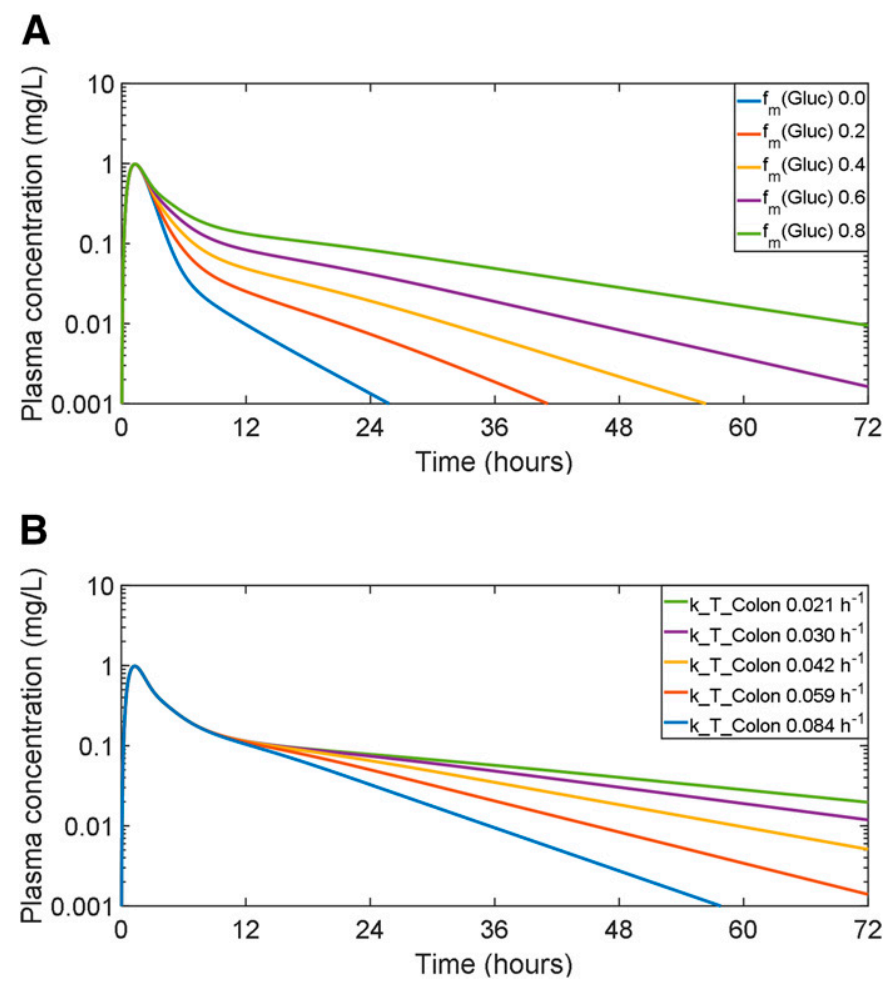

Fig. 7. Sensitivity analyses of effects of $(A) f_{m}($ Gluc) and (B) transit rate in the colon lumen (k_T_Colon) on pharmacokinetic profile of maribavir after a 10-mg/kg single oral administration to cynomolgus monkeys.

terminal half-life and bioavailability; however, the model did not consider the difference between the small and large intestine (radii, transit time, and location of the gut microbiota), nor were any measured drug or metabolite PK data included to validate the model. In our work, the GI tract was compartmentalized with representations of different physiology within each segment; in particular, the colon was separated into five compartments to represent the spatial and temporal transit of both maribavir and its glucuronides. This compartmentalization is a simplification of the continuous transit model of drug-containing intestinal fluid that requires much more complex mathematical methods, but it is also an improvement over models that do not focus on drug absorption and transit in the colon (Yu and Amidon, 1999; Wu, 2012). As a result, the novel semi-PBPK model is able to characterize the segmental (re)absorption of maribavir after either intravenous or p.o. dosing (Supplemental Table 8). Notably, we did not model drug dissolution because of maribavir's aqueous solubility profiles $(0.8 \mathrm{mg} / \mathrm{ml}$ in water; 34 and $0.67 \mathrm{mg} / \mathrm{ml}$ at $\mathrm{pH} 3$ and 6.6, respectively; unpublished data) and because all dosing formulations were in solution; it was also deemed unnecessary to model metabolism of maribavir within the gut wall because of its high permeability and low cytochrome P450-driven intrinsic clearance.

In the semi-PBPK model, four parameters were fitted from the BDC animals' parent PK profiles, whereas all other parameters were either directly from literature or derived from in vitro data or in vivo metabolite data from BDC animals. We then demonstrated that this model captured PK profiles in intact monkeys after single intravenous, single p.o., or multiple p.o. administrations. Through sensitivity analyses, we identified important parameters determining the AUC, $C_{\max }$, and terminal elimination rate for maribavir in plasma. The terminal slope is mostly driven by $\mathrm{f}_{\mathrm{m}}(\mathrm{Gluc})$ and colon transit rate rather than by hepatic clearance, although the latter still drives the overall exposure of maribavir in terms of AUC and $C_{\max }$. In contrast, SITT and luminal hydrolytic rates of the maribavir glucuronides have much smaller impacts on maribavir exposure in intact animals (Supplemental Fig. 29). This semi-PBPK model is applicable to other xenobiotics exhibiting similar phenomena, aiding in comprehension and projection of EHR in preclinical/translational settings; in particular, sensitive drug- and species-related parameters should be given the most attention (Fig. 7; Supplemental Fig. 29).

The $f_{m}$ contributed by each potential clearance pathway is a key drug metabolism and PK metric for small molecular drug candidates because of its implications in the lead optimization process, species translation, clinical study design, and population variability (Di, 2017). For maribavir, it is clear that a higher $\mathrm{f}_{\mathrm{m}}(\mathrm{Gluc})$ will lead to more apparent EHR and higher systemic exposure, as more glucuronides will be generated by hydrolysis in the gut lumen and releasing parent drug for reabsorption. The $\mathrm{f}_{\mathrm{m}}$ (Gluc) for maribavir in monkey was high at $73 \%$ (in vivo) to $85 \%$ (hepatocytes) but only around $20 \%$ in human hepatocytes. Hence, in theory, humans should demonstrate a less significant EHR compared with monkeys. Indeed, in humans, ${ }^{14} \mathrm{C}$-maribavir was primarily eliminated through CYP3A-mediated metabolism with renal clearance as a minor pathway; M4 (VP44469) was the principal metabolite observed in urine and feces (Song et al., 2019b), and no direct glucuronides were detected in feces (unpublished data). No apparent recirculation was observed for plasma profiles of maribavir in humans (Wang et al., 2003), and the $t_{1 / 2}$ observed in humans at around 3.5-7 hours (Wang et al., 2003; Ma et al., 2006; Goldwater et al., 2008; Song et al., 2019a) also mimicked sensitivity analyses wherein $f_{m}$ (Gluc) was changed to $20 \%$ (Fig. 7C), at which scenario the simulated $t_{1 / 2}$ became $\sim 6$ hours (vs. $\sim 15$ hours at $\mathrm{f}_{\mathrm{m}}$ (Gluc) of $80 \%$ ). The much lower $\mathrm{f}_{\mathrm{m}}$ (Gluc) for maribavir in humans thus confers not only a lower risk of drug-drug interaction for increased systemic concentrations of maribavir and its glucuronides when maribavir is coadministered with inhibitors of canalicular efflux transporters (Zamek-Gliszczynski et al., 2014; Patel et al., 2016) or with inhibitors of UGTs (Zhang et al., 2015) but also less risk of reduced systemic concentrations of maribavir due to 
potential induction of UGTs (Court, 2010). In addition, intersubject variabilities in intrinsic factors, such as UGT polymorphism (Court, 2010) and colonic transit time (Vinarov et al., 2021), should have less impact on maribavir exposure in humans due to the lower $\mathrm{f}_{\mathrm{m}}$ (Gluc). Therefore, for drugs that undergo EHR through formation and degradation of conjugative metabolites, species differences in metabolism should be considered when extrapolating PK properties from nonclinical species to humans (Kimoto et al., 2017).

In conclusion, using both intact and bile duct-cannulated animals to measure the metabolism and disposition of maribavir, we quantitatively demonstrated that the primary in vivo clearance pathway of maribavir in monkeys was direct glucuronidation. In intact animals, maribavir undergoes enterohepatic recirculation through biliary secretion of maribavir glucuronides followed by hydrolysis of these glucuronides in the gut lumen and subsequent reabsorption of parent. Finally, the overall pharmacokinetics and disposition of maribavir were mechanistically modeled using semi-physiologically based approaches; the novel model captured maribavir's pharmacokinetic and EHR behavior in intact animals and is indicative of lack of EHR in humans when species differences are incorporated.

\section{Acknowledgments}

The authors would like to acknowledge Aiqun Li, Dan Cui, and Haojing Rong, who at the time of the study were employees of Shire (now Takeda Development Center Americas, Inc.), for their contribution to the experimental studies and Varun Aggarwal, who was an employee of Shire Human Genetic Therapies, Inc. (now Takeda Development Center Americas, Inc.), for technical aspects related to MATLAB and SimBiology. Additionally, the authors thank Matthew Crouthamel, an employee of Takeda Development Center Americas, Inc., and Swapan K. Chowdhury, Cindy Q. Xia, Michael Zientek, and Xiaochun Zhu, who are employees of Takeda, for scientific and editorial inputs during preparation of this manuscript. Additionally, Elizabeth Spencer, Justine Oswald, Sara Leitz, and Mark Gohdes (all employees of Covance, who received funding from Takeda Development Center Americas, Inc., for contracted research) are acknowledged for conducting experiments. Medical writing services were provided by Robert Coover, formerly of Caudex, and Amy Holloway, DPhil, of Caudex, funded by Takeda Development Center Americas, Inc.

\section{Authorship Contributions}

Participated in research design: Sun, Welty.

Conducted experiments: Sun.

Contributed new reagents or analytic tools: Sun.

Performed data analysis: Sun.

Wrote or contributed to the writing of the manuscript: Sun, Welty.

\section{References}

Albert C, Barbier O, Vallée M, Beaudry G, Bélanger A, and Hum DW (2000) Distribution of uridine diphosphate-glucuronosyltransferase (UGT) expression and activity in cynomolgus monkey tissues: evidence for differential expression of steroid-conjugating UGT enzymes in steroid target tissues. Endocrinology 141:2472-2480.

Benson K, Cramer S, and Galla HJ (2013) Impedance-based cell monitoring: barrier properties and beyond. Fluids Barriers CNS 10:5

Biron KK, Harvey RJ, Chamberlain SC, Good SS, Smith 3rd AA, Davis MG, Talarico CL, Miller WH, Ferris R, Dornsife RE, et al. (2002) Potent and selective inhibition of human cytomegalovirus replication by $1263 \mathrm{~W} 94$, a benzimidazole L-riboside with a unique mode of action. Antimicrob Agents Chemother 46:2365-2372.

Court MH (2010) Interindividual variability in hepatic drug glucuronidation: studies into the role of age, sex, enzyme inducers, and genetic polymorphism using the human liver bank as a model system. Drug Metab Rev 42:209-224.

Di L (2017) Reaction phenotyping to assess victim drug-drug interaction risks. Expert Opin Drug Discov 12:1105-1115.

Goldwater DR, Dougherty C, Schumacher M, and Villano SA (2008) Effect of ketoconazole on the pharmacokinetics of maribavir in healthy adults. Antimicrob Agents Chemother 52:1794-1798.
Hakimi Z, Aballéa S, Ferchichi S, Scharn M, Odeyemi IA, Toumi M, and Saliba F (2017) Burden of cytomegalovirus disease in solid organ transplant recipients: a national matched cohort study in an inpatient setting. Transpl Infect Dis 19:e12732.

Hamirally S, Kamil JP, Ndassa-Colday YM, Lin AJ, Jahng WJ, Baek MC, Noton S, Silva LA, Simpson-Holley M, Knipe DM, et al. (2009) Viral mimicry of Cdc2/cyclin-dependent kinase 1 mediates disruption of nuclear lamina during human cytomegalovirus nuclear egress. PLoS Pathog 5:e1000275.

Ikegami K, Tagawa K, Narisawa S, and Osawa T (2003) Suitability of the cynomolgus monkey as an animal model for drug absorption studies of oral dosage forms from the viewpoint of gastrointestinal physiology. Biol Pharm Bull 26:1442-1447.

Khalil F and Läer S (2014) Physiologically based pharmacokinetic models in the prediction of oral drug exposure over the entire pediatric age range-sotalol as a model drug. AAPS J 16:226-239.

Kim DH and Jin YH (2001) Intestinal bacterial beta-glucuronidase activity of patients with colon cancer. Arch Pharm Res 24:564-567.

Kimoto E, Bi YA, Kosa RE, Tremaine LM, and Varma MVS (2017) Hepatobiliary clearance prediction: species scaling from monkey, dog, and rat, and in vitro-in vivo extrapolation of sandwich-cultured human hepatocytes using 17 drugs. J Pharm Sci 106:2795-2804.

Koppel N, Maini Rekdal V, and Balskus EP (2017) Chemical transformation of xenobiotics by the human gut microbiota. Science 356:eaag2770.

Koszalka GW, Johnson NW, Good SS, Boyd L, Chamberlain SC, Townsend LB, Drach JC, and Biron KK (2002) Preclinical and toxicology studies of 1263W94, a potent and selective inhibitor of human cytomegalovirus replication. Antimicrob Agents Chemother 46:2373-2380.

Kotton CN, Kumar D, Caliendo AM, Huprikar S, Chou S, Danziger-Isakov L, and Humar A; The Transplantation Society International CMV Consensus Group (2018) The third international consensus guidelines on the management of cytomegalovirus in solid-organ transplantation. Transplantation 102:900-931.

Krosky PM, Baek MC, and Coen DM (2003) The human cytomegalovirus UL97 protein kinase, an antiviral drug target, is required at the stage of nuclear egress. J Virol 77:905-914.

Ma JD, Nafziger AN, Villano SA, Gaedigk A, and Bertino Jr JS (2006) Maribavir pharmacokinetics and the effects of multiple-dose maribavir on cytochrome P450 (CYP) 1A2, CYP 2C9, CYP 2C19, CYP 2D6, CYP 3A, N-acetyltransferase-2, and xanthine oxidase activities in healthy adults. Antimicrob Agents Chemother 50:1130-1135.

Maertens J, Cordonnier C, Jaksch P, Poiré X, Uknis M, Wu J, Wijatyk A, Saliba F, Witzke O, and Villano S (2019) Maribavir for preemptive treatment of cytomegalovirus reactivation. $N$ Engl J Med 381:1136-1147.

Marty FM, Ljungman P, Chemaly RF, Maertens J, Dadwal SS, Duarte RF, Haider S, Ullmann AJ, Katayama Y, Brown J, et al. (2017) Letermovir prophylaxis for cytomegalovirus in hematopoietic-cell transplantation. $N$ Engl J Med 377:2433-2444.

Matsumoto Y, Cabalu T, Sandhu P, Hartmann G, Iwasa T, Yoshitsugu H, Gibson C, and Uemura N (2019) Application of physiologically based pharmacokinetic modeling to predict pharmacokinetics in healthy Japanese subjects. Clin Pharmacol Ther 105:1018-1030.

National Library of Medicine (2020a) Efficacy and safety study of maribavir compared to valganciclovir for the treatment of cytomegalovirus (CMV) infection in hematopoietic stem cell transplant recipients. https://clinicaltrials.gov/ct2/show/NCT02927067.

National Library of Medicine (2020b) Efficacy and safety study of maribavir treatment compared to investigator-assigned treatment in transplant recipients with cytomegalovirus (CMV) infections that are refractory or resistant to treatment with ganciclovir, valganciclovir, foscarnet, or cidofovir. https://clinicaltrials.gov/ct2/show/NCT02931539.

Papanicolaou GA, Silveira FP, Langston AA, Pereira MR, Avery RK, Uknis M, Wijatyk A, Wu J, Boeckh M, Marty FM, et al. (2019) Maribavir for refractory or resistant cytomegalovirus infections in hematopoietic-cell or solid-organ transplant recipients: a randomized, dose-ranging, double-blind, phase 2 study. Clin Infect Dis 68:1255-1264.

Patel M, Taskar KS, and Zamek-Gliszczynski MJ (2016) Importance of hepatic transporters in clinical disposition of drugs and their metabolites. J Clin Pharmacol 56 (Suppl 7):S23-S39.

Peters SA (2012) Appendix A: physiological parameters in preclinical species, in PhysiologicallyBased Pharmacokinetic (PBPK) Modeling and Simulations: Principles, Methods, and Applications in the Pharmaceutical Industry, pp 408-413, John Wiley \& Sons, Inc., New Jersey.

Sender R, Fuchs S, and Milo R (2016) Revised estimates for the number of human and bacteria cells in the body. PLoS Biol 14:e1002533.

Song I, Ilic K, Sun K, and Martin P (2019a) Clinical pharmacology of maribavir (SHP620): a comprehensive overview, in: Biol Blood Marrow Transplant, pp S342, Transplantation and Cellular Therapy (TCT) Meeting, Houston, TX.

Song I, Sun K, Ilic K, and Martin P (2019b) Summary of maribavir (SHP620) drug-drug interactions based on accumulated clinical and nonclinical data, in: Biol Blood Marrow Transplant, pp S370-S371, Transplantation and Cellular Therapy (TCT) Meeting, Houston, TX

Teira P, Battiwalla M, Ramanathan M, Barrett AJ, Ahn KW, Chen M, Green JS, Saad A, Antin JH, Savani BN, et al. (2016) Early cytomegalovirus reactivation remains associated with increased transplant-related mortality in the current era: a CIBMTR analysis. Blood 127:2427-2438.

Tropini C, Earle KA, Huang KC, and Sonnenburg JL (2017) The gut microbiome: connecting spatial organization to function. Cell Host Microbe 21:433-442.

Venton G, Crocchiolo R, Fürst S, Granata A, Oudin C, Faucher C, Coso D, Bouabdallah R, Berger P, Vey N, et al. (2014) Risk factors of ganciclovir-related neutropenia after allogeneic stem cell transplantation: a retrospective monocentre study on 547 patients. Clin Microbiol Infect 20:160-166.

Vinarov Z, Abdallah M, Agundez JAG, Allegaert K, Basit AW, Braeckmans M, Ceulemans J, Corsetti M, Griffin BT, Grimm M, et al. (2021) Impact of gastrointestinal tract variability on oral drug absorption and pharmacokinetics: an UNGAP review. Eur J Pharm Sci 162:105812.

Wang LH, Peck RW, Yin Y, Allanson J, Wiggs R, and Wire MB (2003) Phase I safety and pharmacokinetic trials of 1263W94, a novel oral anti-human cytomegalovirus agent, in healthy and human immunodeficiency virus-infected subjects. Antimicrob Agents Chemother 47:1334-1342.

Welty D, Sun K, Rong H, and Song I (2018) In vitro metabolic and transporter profiling for marabavir (SHP620), in: 22nd North American ISSX meeting, Montreal, Canada.

Williams SL, Hartline CB, Kushner NL, Harden EA, Bidanset DJ, Drach JC, Townsend LB, Underwood MR, Biron KK, and Kern ER (2003) In vitro activities of benzimidazole D- and Lribonucleosides against herpesviruses. Antimicrob Agents Chemother 47:2186-2192.

Wu B (2012) Use of physiologically based pharmacokinetic models to evaluate the impact of intestinal glucuronide hydrolysis on the pharmacokinetics of aglycone. J Pharm Sci 101:1281-1301.

Yu LX and Amidon GL (1999) A compartmental absorption and transit model for estimating oral drug absorption. Int J Pharm 186:119-125. 
Zamek-Gliszczynski MJ, Chu X, Polli JW, Paine MF, and Galetin A (2014) Understanding the transport properties of metabolites: case studies and considerations for drug development. Drug Metab Dispos 42:650-664.

Zenser TV, Lakshmi VM, and Davis BB (1999) Human and Escherichia coli beta-glucuronidase hydrolysis of glucuronide conjugates of benzidine and 4-aminobiphenyl, and their hydroxy metabolites. Drug Metab Dispos 27:1064-1067.

Zhang N, Liu Y, and Jeong H (2015) Drug-drug interaction potentials of tyrosine kinase inhibitors via inhibition of UDP-glucuronosyltransferases. Sci Rep 5:17778.
Zhou W, Johnson TN, Xu H, Cheung S, Bui KH, Li J, Al-Huniti N, and Zhou D (2016) Predictive performance of physiologically based pharmacokinetic and population pharmacokinetic modeling of renally cleared drugs in children. CPT Pharmacometrics Syst Pharmacol 5:475-483.

Address correspondence to: Kefeng Sun, 300 Shire Way, Lexington, MA 02421. E-mail: kefeng.sun@takeda.com 